\title{
On-line Ramsey Numbers of Paths and Cycles
}

\author{
Joanna Cyman \\ Department of Technical Physics \\ and Applied Mathematics, \\ Gdańsk University of Technology, \\ Narutowicza 11/12, 80-952 Gdańsk, \\ Poland \\ joana@mif.pg.gda.pl \\ John Lapinskas ${ }^{\dagger}$ \\ Department of Computer Science, \\ University of Oxford, \\ Wolfson Building, Parks Road, \\ Oxford, OX1 3QD, \\ United Kingdom \\ lapinskas@cs.ox.ac.uk
}

\author{
Tomasz Dzido* \\ Institute of Informatics, \\ University of Gdańsk, \\ Wita Stwosza 57, 80-952 Gdańsk, \\ Poland \\ tdz@inf.ug.edu.pl
}

Submitted: Feb 14, 2014; Accepted: Jan 13, 2015; Published: Jan 20, 2015

Mathematics Subject Classifications: 05C55, 05C57, 05C15, 91A46

\begin{abstract}
Consider a game played on the edge set of the infinite clique by two players, Builder and Painter. In each round, Builder chooses an edge and Painter colours it red or blue. Builder wins by creating either a red copy of $G$ or a blue copy of $H$ for some fixed graphs $G$ and $H$. The minimum number of rounds within which Builder can win, assuming both players play perfectly, is the on-line Ramsey number $\tilde{r}(G, H)$. In this paper, we consider the case where $G$ is a path $P_{k}$. We prove that $\tilde{r}\left(P_{3}, P_{\ell+1}\right)=\lceil 5 \ell / 4\rceil=\tilde{r}\left(P_{3}, C_{\ell}\right)$ for all $\ell \geqslant 5$, and determine $\tilde{r}\left(P_{4}, P_{\ell+1}\right)$ up to an additive constant for all $\ell \geqslant 3$. We also prove some general lower bounds for on-line Ramsey numbers of the form $\tilde{r}\left(P_{k+1}, H\right)$.
\end{abstract}

Keywords: On-line Ramsey theory; Combinatorial games; Paths; Cycles.

*Partially supported by the Polish National Science Centre grant 2011/02/A/ST6/00201.

${ }^{\dagger}$ The research leading to these results has received funding from the European Research Council under the European Union's Seventh Framework Programme (FP7/2007-2013) ERC grant agreements no. 334828. The paper reflects only the authors' views and not the views of the ERC or the European Commission. The European Union is not liable for any use that may be made of the information contained therein.

¥The research leading to these results has received funding from the European Research Council under the European Union's Seventh Framework Programme (FP7/2007-2013) ERC grant agreements no. 258345 . 


\section{Introduction}

Ramsey's theorem [16] states that for all $k \in \mathbb{N}$, there exists $t \in \mathbb{N}$ such that any red-blue edge colouring of a clique $K_{t}$ contains a monochromatic clique of order $k$. We call the least such $t$ the $k^{\text {th }}$ Ramsey number, and denote it by $r(k)$. Ramsey numbers and their generalisations have been a fundamentally important area of study in combinatorics for many years. Particularly well-studied are Ramsey numbers for graphs. Here the Ramsey number of two graphs $G$ and $H$, denoted by $r(G, H)$, is the least $t$ such that any red-blue edge colouring of $K_{t}$ contains a red copy of $G$ or a blue copy of $H$. See e.g. [15] for a survey of known Ramsey numbers.

An important generalisation of Ramsey numbers, first defined by Erdős, Faudree, Rousseau and Schelp [5], is as follows. Let $G$ and $H$ be two graphs. We say that a graph $K$ has the $(G, H)$-Ramsey property if any red-blue edge colouring of $K$ must contain either a red copy of $G$ or a blue copy of $H$. Then the size Ramsey number $\hat{r}(G, H)$ is given by the minimum number of edges of any graph with the $(G, H)$-Ramsey property.

In this paper, we consider the following related generalisation defined independently by Beck [1] and Kurek and Ruciński [10]. Let $G$ and $H$ be two graphs. Consider a game played on the edge set of the infinite clique $K_{\mathbb{N}}$ with two players, Builder and Painter. In each round of the game, Builder chooses an edge and Painter colours it red or blue. Builder wins by creating either a red copy of $G$ or a blue copy of $H$, and wishes to do so in as few rounds as possible. Painter wishes to delay Builder for as many rounds as possible. (Note that Painter may not delay Builder indefinitely - for example, Builder may simply choose every edge of $K_{r(G, H)}$.) The on-line Ramsey number $\tilde{r}(G, H)$ is the minimum number of rounds it takes Builder to win, assuming that both Builder and Painter play optimally. We call this game the $\tilde{r}(G, H)$-game, and write $\tilde{r}(G)=\tilde{r}(G, G)$. Note that $\tilde{r}(G, H) \geqslant e(G)+e(H)-1$ for all graphs $G$ and $H$, as Painter may simply colour the first $e(G)-1$ edges red and all subsequent edges blue. It is also clear that $\tilde{r}(G, H) \leqslant \hat{r}(G, H)$.

On-line Ramsey theory has been well-studied. The best known bounds for $\tilde{r}\left(K_{t}\right)$ are given by

$$
\frac{r(t)-1}{2} \leqslant \tilde{r}\left(K_{t}\right) \leqslant t^{-c \frac{\log t}{\log \log t} 4^{t}},
$$

where $c$ is a positive constant. The lower bound is due to Alon (and was first published in a paper of Beck [1]), and the upper bound is due to Conlon [3]. Note that these bounds are similar to the best known bounds for classical Ramsey numbers $r(t)$, although Conlon also proves in [3] that

$$
\tilde{r}\left(K_{t}\right) \leqslant C^{-t}\left(\begin{array}{c}
r(t) \\
2
\end{array}\right)
$$

for some constant $C>1$ and infinitely many values of $t$, which gives positive evidences supporting a conjecture of Kurek and Ruciński [10] that $\tilde{r}\left(K_{t}\right)=o\left(r(t)^{2}\right)$. For general graphs $G$, the best known lower bound for $\tilde{r}(G)$ is given by Grytczuk, Kierstead and Prałat [8]. 
Theorem 1. For graphs $G$, we have $\tilde{r}(G) \geqslant \beta(G)(\Delta(G)-1) / 2+e(G)$, where $\beta(G)$ denotes the vertex cover number of $G$.

Various general strategies for Builder and Painter have also been studied. For example, consider the following strategy for Builder in the $\tilde{r}(G, H)$-game. Builder chooses a large but finite set of vertices in $K_{\mathbb{N}}$, say a set of size $n \in \mathbb{N}$, with $n \geqslant r(G, H)$. Then Builder chooses the edges of the induced $K_{n}$ in a uniformly random order, allowing Painter to colour each edge as they wish, until the game ends. This strategy was analysed for the $\tilde{r}\left(K_{3}\right)$-game by Friedgut, Kohayakawa, Rödl, Ruciński and Tetali [6], and for the more general $\tilde{r}(G)$-game by Marciniszyn, Spöhel and Steger [11, 12].

Finally, let $\tilde{r}_{\chi}(G)$-game be the $\tilde{r}(G)$-game in which Builder is forbidden to uncovering a graph with chromatics number greater than $\chi(G)$. Grytczuk, Hałuszczak and Kierstead [7] proved that Builder can win the $\tilde{r}_{\chi}(G)$-game. Kierstead and Konjevod [9] proved the hypergraph generalisation.

Given the known bounds on $\tilde{r}\left(K_{t}\right)$, it is not surprising that determining on-line Ramsey numbers exactly has proved even more difficult than determining classical Ramsey numbers exactly, and very few results are known. A significant amount of effort has been focused on the special case where $G$ and $H$ are paths. Grytczuk, Kierstead and Prałat [8] and Prałat $[13,14]$ have determined $\tilde{r}\left(P_{k+1}, P_{\ell+1}\right)$ exactly when $\max \{k, \ell\} \leqslant 8$ (where $P_{s}$ is a path on $s$ verices). In addition, Beck [2] has proved that the size Ramsey number $\hat{r}\left(P_{k}, P_{k}\right)$ is linear in $k$. (The best known upper bound, due to Dudek and Prałat [4], is $\hat{r}\left(P_{k}, P_{k}\right) \leqslant 137 k$. $)$ The best known bounds on $\tilde{r}\left(P_{k+1}, P_{\ell+1}\right)$ were proved in [8].

Theorem 2. For all $k, \ell \in \mathbb{N}$, we have $k+\ell-1 \leqslant \tilde{r}\left(P_{k+1}, P_{\ell+1}\right) \leqslant 2 k+2 \ell-3$.

In general, it seems difficult to bound on-line Ramsey numbers $\tilde{r}(G, H)$ below. One of the major difficulties in doing so is the variety of possible strategies for Builder. We present a strategy for Painter which mitigates this problem somewhat.

Definition 3. Let $\mathcal{F}$ be a family of graphs. We define the $\mathcal{F}$-blocking strategy for Painter as follows. Write $R_{i}$ for the graph consisting of all uncovered red edges immediately before the $i$ th move of the game, and write $e_{i}$ for the $i$ th edge chosen by Builder. Then Painter colours $e_{i}$ red if $R_{i}+e_{i}$ is $\mathcal{F}$-free, and blue otherwise. (Recall that a graph is $\mathcal{F}$-free if it contains no graph in $\mathcal{F}$ as a subgraph.)

In an $\tilde{r}(G, H)$-game, it is natural to consider $\mathcal{F}$-blocking strategies with $G \in \mathcal{F}$. For example, if $\mathcal{F}=\{G\}$, then the $\mathcal{F}$-blocking strategy for Painter consists of colouring every edge red unless doing so would cause Painter to lose the game. If Painter is using an $\mathcal{F}$-blocking strategy, one clear strategy for Builder would be to construct a red $\mathcal{F}$-free graph, then use it to force a blue copy of $H$ in $e(H)$ moves. We will show that this is effectively Builder's only strategy (see Proposition 13 ), and thus to bound $\tilde{r}(G, H)$ below it suffices to prove that no small red $\mathcal{F}$-free graph can be used to force a blue copy of $H$. We use this technique to derive some lower bounds for on-line Ramsey numbers of the form $\tilde{r}\left(P_{k+1}, H\right)$, taking $\mathcal{F}=\left\{P_{k+1}\right\} \cup\left\{C_{i}: i \geqslant 3\right\}$. 
Theorem 4. Let $k, \ell \in \mathbb{N}$ with $k \geqslant 2$. Let $H$ be a graph on $|H|$ vertices with $\ell$ edges and let $\Delta=\Delta(H)$. Then

$$
\tilde{r}\left(P_{k+1}, H\right) \geqslant \begin{cases}(2 \Delta+1) \ell /(2 \Delta) & \text { if } k=2 \\ (5 \Delta+4) \ell /(5 \Delta) & \text { if } k=3 \\ (\Delta+1) \ell / \Delta & \text { if } k \geqslant 4\end{cases}
$$

Moreover, if $H$ is connected and $k \geqslant 4$, then

$$
\tilde{r}\left(P_{k+1}, H\right) \geqslant(\Delta+1) \ell / \Delta+\min \{k / 2-2,|H|-1\} .
$$

For $k=2$, we show that if $H=P_{\ell+1}$ for $\ell \geqslant 2$ or $H=C_{\ell}$ for $\ell \geqslant 5$, then the bound on $\tilde{r}\left(P_{3}, H\right)$ given by Theorem 4 is tight.

Theorem 5. For all $\ell \geqslant 2$, we have $\tilde{r}\left(P_{3}, P_{\ell+1}\right)=\lceil 5 \ell / 4\rceil$. Also,

$$
\tilde{r}\left(P_{3}, C_{\ell}\right)= \begin{cases}\ell+2 & \text { if } \ell=3,4 \\ \lceil 5 \ell / 4\rceil & \text { if } \ell \geqslant 5 .\end{cases}
$$

Furthermore, for $k=3$, we determine $\tilde{r}\left(P_{4}, P_{\ell+1}\right)$ up to an additive constant for all $\ell \geqslant 3$.

Theorem 6. For all $\ell \geqslant 3$, we have $(7 \ell+2) / 5 \leqslant \tilde{r}\left(P_{4}, P_{\ell+1}\right) \leqslant(7 \ell+52) / 5$.

Our proof of the upper bound for $k=3$ is complicated, so the proof is included in the Appendix. The lower bound follows from Lemma 18, a simple extension of the proof of Theorem 4, and we believe that it is tight.

Conjecture 7. For all $\ell \geqslant 3$, we have $\tilde{r}\left(P_{4}, P_{\ell+1}\right)=\lceil(7 \ell+2) / 5\rceil$.

By Theorems 5 and 6 , we have

$$
\begin{gathered}
\lim _{\ell \rightarrow \infty} \tilde{r}\left(P_{3}, P_{\ell+1}\right) / \ell=5 / 4, \\
\lim _{\ell \rightarrow \infty} \tilde{r}\left(P_{4}, P_{\ell+1}\right) / \ell=7 / 5 .
\end{gathered}
$$

On the other hand, for all fixed $k \geqslant 4$, Theorems 2 and 4 imply that

$$
3 / 2 \leqslant \liminf _{\ell \rightarrow \infty} \tilde{r}\left(P_{k+1}, P_{\ell+1}\right) / \ell \leqslant \limsup _{\ell \rightarrow \infty} \tilde{r}\left(P_{k+1}, P_{\ell+1}\right) / \ell \leqslant 2,
$$

and we make the following conjecture.

Conjecture 8. For $k \geqslant 4, \lim _{\ell \rightarrow \infty} \tilde{r}\left(P_{k+1}, P_{\ell+1}\right) / \ell=3 / 2$. Moreover, for all $\ell \geqslant k \geqslant 4$, we have $\tilde{r}\left(P_{k+1}, P_{\ell+1}\right)=\lceil 3 \ell / 2\rceil+k-3$. In particular, we have $\tilde{r}\left(P_{k+1}\right)=\lceil 5 k / 2\rceil-3$ for $k \geqslant 4$. 
Note that Conjecture 8 would imply Conjecture 4.1 of [14]. Conjectures 7 and 8 have been confirmed for $\ell \leqslant 8$ by Prałat [13], using a high-performance computer cluster.

Finally, we give some bounds on $\tilde{r}\left(C_{4}, P_{\ell+1}\right)$.

Theorem 9. For $\ell \geqslant 3$, we have $2 \ell \leqslant \tilde{r}\left(C_{4}, P_{\ell+1}\right) \leqslant 4 \ell-4$. Moreover, $\tilde{r}\left(C_{4}, P_{4}\right)=8$.

Many of the lower bounds above follow from Theorem 4, and all of them follow from analysing $\mathcal{F}$-blocking strategies. In particular, we obtain tight lower bounds on $\tilde{r}\left(P_{3}, P_{\ell+1}\right)$ and $\tilde{r}\left(P_{3}, C_{\ell}\right)$ in this way, as well as a lower bound on $\tilde{r}\left(P_{4}, P_{\ell+1}\right)$ which matches Conjecture 7 . We are therefore motivated to ask the following question.

Question 10. For which graphs $G$ and $H$ does there exist a family $\mathcal{F}$ of graphs such that the $\mathcal{F}$-blocking strategy is optimal for Painter in the $\tilde{r}(G, H)$-game?

The paper is laid out as follows. In Section 3, we prove Theorem 4. We prove Theorem 5 in Sections 4 and 5 (see Theorem 21, Proposition 23 and Theorem 24). Finally, in Section 6 we prove Theorem 9. The proof of Theorem 6 is in the Appendix.

\section{Notation and conventions}

We write $\mathbb{N}$ for the set $\{1,2, \ldots\}$ of natural numbers, and $\mathbb{N}_{0}:=\mathbb{N} \cup\{0\}$.

Suppose $P=v_{1} \ldots v_{k}$ and $Q=w_{1} \ldots w_{\ell}$ are paths. If $i<j$, we write $v_{i} P v_{j}$ (or $\left.v_{j} P v_{i}\right)$ for the subpath $v_{i} v_{i+1} \ldots v_{j}$ of $P$. We also write $P Q$ for the concatenation of $P$ and $Q$. For example, if $i<j$ and $i^{\prime}<j^{\prime}$ then $u v_{i} P v_{j} y w_{i^{\prime}} Q w_{j^{\prime}}$ denotes the path $u v_{i} v_{i+1} \ldots v_{j} y w_{i^{\prime}} w_{i^{\prime}+1} \ldots w_{j^{\prime}}$.

If $G$ is a graph, we will write $|G|$ for the number of vertices of $G$ and $e(G)$ for the number of edges of $G$.

In the context of an $\tilde{r}(G, H)$-game, an uncovered edge is an edge of $K_{\mathbb{N}}$ that has previously been chosen by Builder, and a new vertex is a vertex in $K_{\mathbb{N}}$ not incident to any uncovered edge.

Many of our lemmas say that in an $\tilde{r}(G, H)$-game, given a finite coloured graph $X \subseteq$ $K_{\mathbb{N}}$, Builder can force Painter to construct a coloured graph $Y \subseteq K_{\mathbb{N}}$ satisfying some desired property. We will often apply such a lemma to a finite coloured graph $X^{\prime} \supsetneq X$, and in these cases we will implicitly require $V(Y) \cap V\left(X^{\prime}\right) \subseteq V(X)$. (Intuitively, when Builder chooses a new vertex while constructing $Y$, it should be new with respect to $X^{\prime}$ rather than $X$.) This is formally valid, since we may apply the lemma to an $\tilde{r}(G, H)$ game on the board $K_{\mathbb{N}}-\left(V\left(X^{\prime}\right) \backslash V(X)\right)$ and have Builder choose the corresponding edges in $K_{\mathbb{N}}$.

For technical convenience, we allow Builder to "waste" a round in the $\tilde{r}(G, H)$-game by choosing an uncovered edge. If he does so, the round contributes to the duration of the game but the edge Builder chooses is not recoloured. Since such a move is never optimal for Builder, the definition of $\tilde{r}(G, H)$ is not affected. 


\section{General lower bounds}

Our aim is to bound $\tilde{r}(G, H)$ below for graphs $G$ and $H$. In this section, Painter will always use an $\mathcal{F}$-blocking strategy for some family $\mathcal{F}$ of graphs with $G \in \mathcal{F}$. Hence, as we shall demonstrate in Proposition 13 below, Builder's strategy boils down to choosing a red graph with which to force a blue copy of $H$.

Definition 11. Let $\mathcal{F}$ be a family of graphs and let $R \subseteq K_{\mathbb{N}}$ be an $\mathcal{F}$-free graph. We say that an edge $e \in K_{\mathbb{N}}-R$ is $(R, \mathcal{F})$-forceable if $R+e$ is not $\mathcal{F}$-free. We say a graph $H$ is $(R, \mathcal{F})$-forceable if there exists $H^{\prime} \subseteq K_{\mathbb{N}}-R$ with $H^{\prime}$ isomorphic to $H$ such that every edge $e \in E\left(H^{\prime}\right)$ is $(R, \mathcal{F})$-forceable. We call $H^{\prime}$ an $(R, \mathcal{F})$-forced copy of $H$. If $R$ and $\mathcal{F}$ are clear from context, we will omit ' $(R, \mathcal{F})-$ '.

Definition 12. Let $\mathcal{F}$ be a family of graphs and let $H$ be a graph. We say a graph $R \subseteq K_{\mathbb{N}}$ is an $\mathcal{F}$-scaffolding for $H$ if the following properties hold.

(i) $R$ is $\mathcal{F}$-free.

(ii) $H$ is $(R, \mathcal{F})$-forceable.

(iii) $R$ contains no isolated vertices.

Proposition 13. Let $G$ and $H$ be graphs. Let $\mathcal{F}$ be a family of graphs with $G \in \mathcal{F}$. Suppose every $\mathcal{F}$-scaffolding for $H$ has at least $m$ edges. Then $\tilde{r}(G, H) \geqslant m+e(H)$.

Proof. Consider an $\tilde{r}(G, H)$-game in which Painter uses an $\mathcal{F}$-blocking strategy. Further suppose Builder wins by claiming edges $e_{1}, \ldots, e_{r}$. Since Builder choosing an edge which Painter colours blue has no effect on Painter's subsequent choices, without loss of generality we may assume that there exists $i$ such that Painter colours $e_{1}, \ldots, e_{i}$ red and $e_{i+1}, \ldots, e_{r}$ blue. Let $R \subseteq K_{\mathbb{N}}$ be the subgraph with edge set $\left\{e_{1}, \ldots, e_{i}\right\}$, and let $B \subseteq K_{\mathbb{N}}$ be the subgraph with edge set $\left\{e_{i+1}, \ldots, e_{r}\right\}$. Thus $R$ is the uncovered red graph and $B$ is the uncovered blue graph.

We will show that $R$ is an $\mathcal{F}$-scaffolding for $H$. First note that $R$ is $\mathcal{F}$-free by Painter's strategy, and $R$ has no isolated vertices by definition. Moreover, since $G \in \mathcal{F}$ and Builder wins, there exists $H^{\prime} \subseteq B$ with $H^{\prime}$ isomorphic to $H$. So $e(B) \geqslant e(H)$. Moreover, by Painter's strategy all edges in $B$ must be $(R, \mathcal{F})$-forceable, so $H$ is $(R, \mathcal{F})$-forceable. Hence $R$ is an $\mathcal{F}$-scaffolding for $H$, so $e(R) \geqslant m$. Therefore, Builder wins in $r \geqslant e(R)+e(B) \geqslant$ $m+e(H)$ rounds.

Therefore, to bound $\tilde{r}(G, H)$ below, it suffices to bound the number of edges in an $\mathcal{F}$-scaffolding for $H$ below for some family $\mathcal{F}$ of graphs with $G \in \mathcal{F}$. We first use Proposition 13 to bound $\tilde{r}\left(C_{k}, H\right)$ for connected graphs $H$.

Lemma 14. Let $H$ be a connected graph. Then every $\left\{C_{i}: i \geqslant 3\right\}$-scaffolding for $H$ has at least $|H|-1$ edges. Moreover, $\tilde{r}\left(C_{k}, H\right) \geqslant|H|+e(H)-1$ for all $k \geqslant 3$. 
Proof. Let $R$ be a $\left\{C_{k}\right\}$-scaffolding for $H$ with $e(R)$ minimal. Note that each $\left(R,\left\{C_{k}\right\}\right)$ forceable edge must lie entirely in a component of $R$. Since $H$ is connected, $R$ is connected and $|R| \geqslant|H|$. Hence, $e(R) \geqslant|H|-1$.

By Proposition 13, $\tilde{r}\left(C_{k}, H\right) \geqslant|H|+e(H)-1$.

To prove Theorem 4 , we set $G=P_{k+1}$ and $\mathcal{F}=\left\{P_{k+1}\right\} \cup\left\{C_{i}: i \geqslant 3\right\}$. Thus an $\mathcal{F}$-free graph is a forest whose components have diameter less than $k$. Lemma 17 gives a lower bound on the number of edges in an $\mathcal{F}$-scaffolding for $H$.

Note that replacing $\mathcal{F}$ by $\left\{P_{k+1}\right\}$ and attempting a similar proof yields a worse lower bound in some cases. For example, taking $H=P_{2 k+1}$ with $k \geqslant 3$, if Painter follows the $\left\{P_{k+1}\right\}$-blocking strategy then Builder can win in $3 k$ moves by first constructing a red $C_{k}$.

We will see in the proof of Lemma 17 that if $R$ is a red $\mathcal{F}$-free graph with no isolated vertices, and $X \subseteq V(R)$ is the set of endpoints of $P_{k}$ 's in $R$, then Builder may force at most $\Delta(H)(|R|+|X|)$ edges of $H$ using $R$. It will therefore be very useful to bound $|R|+|X|$ above in terms of $e(R)$, first in the special case where $R$ is a tree (see Lemma 15) and then in general (see Lemma 16).

Lemma 15. Let $k, m \in \mathbb{N}$ with $k \geqslant 2$. Let $R$ be a $P_{k+1}$-free tree with $m$ edges. Let $X$ be the set of endpoints of $P_{k}$ 's in $R$. If $X \neq \emptyset$, then $|R|+|X| \leqslant 2 m-k+4$.

Proof. We claim that if $x \in X$, then $x$ is a leaf of $R$. Indeed, let $P$ be a $P_{k}$ with one endpoint equal to $x$. Let $y \in V(P)$ be the neighbour of $x$ in $P$, and suppose $x z \in E(R)$ for some $z \neq y$. Then either $z \in V(P)$ and $x z P x$ is a cycle in $R$, or $z \notin V(P)$ and $P x z$ is a $P_{k+1}$ in $R$ - both are contradictions. Hence if $x \in X$, then $x$ is a leaf. But since $X \neq \emptyset$, $R$ contains a $P_{k}$ and hence at least $k-2$ vertices of degree greater than 1 . Hence

$$
|R|+|X| \leqslant|R|+|R|-(k-2)=2 m-k+4,
$$

and the proposition follows.

Lemma 16. Let $k, m \in \mathbb{N}$ with $k \geqslant 2$. Let $R$ be a $P_{k+1}$-free forest with $m$ edges and no isolated vertices. Let $X$ be the set of all endpoints of $P_{k}$ 's in $R$. Then

$$
|R|+|X| \leqslant \begin{cases}4 m & \text { if } k=2 \\ 5 m / 2 & \text { if } k=3 \\ 2 m & \text { if } k \geqslant 4\end{cases}
$$

Moreover, if $k \geqslant 4$ and there exists an edge $e$ such that $R+e$ contains a $P_{k+1}$, then $|R|+|X| \leqslant 2 m-k+4$.

Proof. Let $R_{1}, \ldots, R_{r}$ be the components of $R$. Let $m_{i}=e\left(R_{i}\right)$ and $X_{i}=X \cap V\left(R_{i}\right)$ for all $1 \leqslant i \leqslant r$. If $k=2$, then $R$ is a disjoint union of $m$ edges and the result is immediate.

Suppose $k=3$. Without loss of generality, let $R_{1}, \ldots, R_{r^{\prime}}$ be those components of $R$ which consist of a single edge. (Note that we may have $r^{\prime}=0$.) Then $m=r^{\prime}+\sum_{i=r^{\prime}+1}^{r} m_{i}$ 
and $r-r^{\prime} \leqslant m / 2$. Then by Lemma 15 we have

$$
\begin{aligned}
|R|+|X| & =\sum_{i=1}^{r^{\prime}}\left|R_{i}\right|+\sum_{i=r^{\prime}+1}^{r}\left(\left|R_{i}\right|+\left|X_{i}\right|\right) \leqslant 2 r^{\prime}+\sum_{i=r^{\prime}+1}^{r}\left(2 m_{i}+1\right) \\
& =2 m+r-r^{\prime} \leqslant 5 m / 2
\end{aligned}
$$

and so the result follows.

Finally, suppose $k \geqslant 4$. Let $q$ be the number of components of $R$ containing a $P_{k}$. Without loss of generality suppose that $R_{1}, \ldots, R_{q}$ are the components of $R$ which contain a $P_{k}$. For $q<i \leqslant r$, we have $\left|R_{i}\right|+\left|X_{i}\right|=\left|R_{i}\right|=m_{i}+1 \leqslant 2 m_{i}$. Then by Lemma 15 we have

$$
|R|+|X|=\sum_{i=1}^{r}\left(\left|R_{i}\right|+\left|X_{i}\right|\right) \leqslant \sum_{i=1}^{q}\left(2 m_{i}-k+4\right)+\sum_{i=q+1}^{r}\left(2 m_{i}\right)=2 m-q(k-4) .
$$

Suppose that there exists an edge $e$ such that $R+e$ contains a $P_{k+1}$. If $X \neq \emptyset$, then $q \geqslant 1$ and so $|R|+|X| \leqslant 2 m-k+4$ by (1). Hence we may assume that $X=\emptyset$, and so $e$ is an edge between two vertices of $R$. It follows that $R$ contains two vertex-disjoint paths of combined length at least $k-1$, and hence that

$$
|R|+|X|=|R|=m+r \leqslant m+(m-k+3)<2 m-k+4,
$$

as desired. The first inequality follows since all edges in a given path must lie in the same component of $R$.

Lemma 17. Let $k, \ell \in \mathbb{N}$ with $k \geqslant 2$. Let $H$ be a graph with $\ell$ edges and let $\Delta=\Delta(H)$. Let $\mathcal{F}=\left\{P_{k+1}\right\} \cup\left\{C_{i}: i \geqslant 3\right\}$. Suppose $R$ is an $\mathcal{F}$-scaffolding for $H$. Then, we have

$$
e(R) \geqslant \begin{cases}\ell /(2 \Delta) & \text { if } k=2 \\ 4 \ell /(5 \Delta) & \text { if } k=3 \\ \ell / \Delta & \text { if } k \geqslant 4\end{cases}
$$

Moreover, if $H$ is connected and $k \geqslant 4$ then $e(R) \geqslant \min \left\{\frac{\ell}{\Delta}+\frac{k}{2}-2,|H|-1\right\}$.

Proof. Let $m=e(R)$. Note that $R$ is a $P_{k+1}$-free forest with $m$ edges and no isolated vertices. Let $X$ be the set of endpoints of $P_{k}$ 's in $R$ and let $Y=V(R) \backslash X$.

We first claim that any $(R, \mathcal{F})$-forceable edge is either incident to $X$ or internal to $Y$. Suppose not. Then there exist $y \in Y$ and $z \notin V(R)$ such that $y z$ is a forceable edge. Let $F \in \mathcal{F}$ be such that $F \subseteq R+e$. Note that $e \in E(F)$, since $R$ is $\mathcal{F}$-free. Since $d_{R+e}(z)=1$, we have $F=P_{k+1}$. But then $y$ is an endpoint of a $P_{k}$ in $R$, contradicting $y \in Y$.

Let $H^{\prime}$ be a forced copy of $H$. Then $H^{\prime}$ contains at most $\Delta|X|$ edges incident to $X$, and at most $\Delta|Y| / 2$ edges internal to $Y$. All edges of $H^{\prime}$ are forceable, so it follows that

$$
\ell=e\left(H^{\prime}\right) \leqslant \Delta|X|+\frac{\Delta|Y|}{2}=\frac{\Delta(|R|+|X|)}{2} .
$$


Lemma 16 and (2) imply the lemma holds unless $k \geqslant 4$ and $H$ is connected.

Now suppose $H$ is connected and $k \geqslant 4$. If there exists an edge $e$ such that $R+e$ contains a $P_{k+1}$, then $|R|+|X| \leqslant 2 m-k+4$ by Lemma 16. Hence, (2) implies that $m \geqslant \frac{\ell}{\Delta}+\frac{k}{2}-2$. Therefore, we may assume that no such edge exists, and in particular that $X=\emptyset$. This implies that $R$ is a $\left\{C_{i}: i \geqslant 3\right\}$-scaffolding for $H$. Lemma 14 implies that $m \geqslant|H|-1$ as required.

Theorem 4 follows immediately from Proposition 13 and Lemma 17.

We now bound $\tilde{r}\left(P_{4}, P_{\ell+1}\right)$ from below.

Lemma 18. Let $\ell \in \mathbb{N}$ with $\ell \geqslant 3$. Then we have $\tilde{r}\left(P_{4}, P_{\ell+1}\right) \geqslant(7 \ell+2) / 5$.

Proof. Let $\mathcal{F}=\left\{P_{4}\right\} \cup\left\{C_{i}: i \geqslant 3\right\}$. Let $R$ be an $\mathcal{F}$-scaffolding for $P_{\ell+1}$. Let $X$ be the set of endpoints of $P_{3}$ 's in $R$, and let $Y=V(R) \backslash X$. By Lemma 16 and Proposition 13, to prove the lemma it suffices to show that $|R|+|X| \geqslant \ell+1$.

Let $H$ be a forced copy of $P_{\ell+1}$. Note that any $(R, \mathcal{F})$-forceable edge is either incident to $X$ or internal to $Y$. Note also that $Y \neq \emptyset$. Indeed, if $X=\emptyset$ then this is immediate. If $X \neq \emptyset$, then $R$ is a $P_{4}$-free forest containing a $P_{3}$. The central vertex of this $P_{3}$ cannot be an element of $X$, and is therefore an element of $Y$.

Since $\Delta(H)=2, H$ contains at most $2|X|$ edges incident to $X$. Moreover, since $H$ is a path, $H[Y]$ is a forest and so $e_{H}(Y) \leqslant|Y|-1$. It follows that

$$
\ell \leqslant 2|X|+|Y|-1=|R|+|X|-1
$$

and hence $|R|+|X| \geqslant \ell+1$ as desired.

\section{Determining $\tilde{r}\left(P_{3}, P_{\ell+1}\right)$ for $\ell \geqslant 2$}

Theorem 4 implies that $\tilde{r}\left(P_{3}, P_{\ell+1}\right) \geqslant\lceil 5 \ell / 4\rceil$ for $\ell \geqslant 2$. To bound $\tilde{r}\left(P_{3}, P_{\ell+1}\right)$ above, we shall present a strategy for Builder. In the discussion that follows, we assume for clarity that Painter will never voluntarily lose the $\tilde{r}\left(P_{3}, P_{\ell+1}\right)$-game.

Builder will use the threat of a red $P_{3}$ to force a blue $P_{\ell+1}$. First, Builder will use Lemma 19 to construct a blue path $P$ with one endpoint incident to a red edge. Builder will then use a procedure outlined in Lemma 20 to efficiently extend $P$ until it has length between $\ell-4$ and $\ell$. Finally, Builder will carefully extend $P$ into a blue $P_{\ell+1}$, yielding a tight upper bound for $\tilde{r}\left(P_{3}, P_{\ell+1}\right)$ (see Theorem 21).

Lemma 19. Let $q \in \mathbb{N}$ with $q \geqslant 5$. Builder can force one of the following structures independent of Painter's choices:

(i) a red $P_{3}$ in at most $q-1$ rounds.

(ii) a blue $P_{q}$ in $q-1$ rounds. 
(iii) a blue $P_{t}$ with one endpoint incident to a red edge in $t$ rounds for some $4 \leqslant t \leqslant q-1$.

Proof. Builder first chooses an arbitrary vertex $x_{1}$, then proceeds as follows. Suppose that Builder has already obtained a blue path $x_{1} \ldots x_{i}$ in $i-1$ rounds for some $1 \leqslant i<q$. Builder then chooses the edge $x_{i} x_{i+1}$, where $x_{i+1}$ is a new vertex. If Painter colours $x_{i} x_{i+1}$ blue, we have obtained a blue path $x_{1} \ldots x_{i+1}$ in $i$ rounds, and so if $i+1<q$ we may repeat the process. If Painter colours all such edges blue, we will obtain a blue path $x_{1} \ldots x_{q}$ in $q-1$ rounds and achieve (ii). Suppose instead that for some $1 \leqslant i \leqslant q-1$, within $i$ rounds we obtain a path $x_{1} \ldots x_{i+1}$ such that $x_{1} \ldots x_{i}$ is blue and $x_{i} x_{i+1}$ is red. If $i \geqslant 4$ then we have achieved (iii), so suppose in addition $i \leqslant 3$.

First suppose $i \in\{1,2\}$. In this case, Builder chooses the two edges $x_{i} v$ and $v x_{i+1}$ where $v$ is a new vertex. If $i=1$, Builder also chooses the edge $x_{i+1} w$ where $w$ is a new vertex. If Painter colours $x_{i} v, v x_{i+1}$ or $x_{i+1} w$ red, then $x_{i+1} x_{i} v, v x_{i+1} x_{i}$ or $x_{i} x_{i+1} w$ respectively is a red $P_{3}$ and we have achieved (i). Otherwise, we have achieved (iii). Indeed, if $i=1$ then $x_{1} v x_{2} w$ is a blue $P_{4}$ constructed in 4 rounds with $x_{1}$ incident to the red edge $x_{1} x_{2}$, and if $i=2$ then $x_{1} x_{2} v x_{3}$ is a blue $P_{4}$ constructed in 4 rounds with $x_{3}$ incident to the red edge $x_{3} x_{2}$.

Finally, suppose $i=3$. Then Builder chooses the edge $x_{4} x_{1}$. If Painter colours the edge red, then $x_{3} x_{4} x_{1}$ is a red $P_{3}$ and we have achieved (i), so suppose Painter colours the edge blue. Then $x_{4} x_{1} x_{2} x_{3}$ is a blue $P_{4}$ constructed in 4 rounds with $x_{3}$ incident to the red edge $x_{3} x_{4}$, so we have achieved (iii).

Lemma 20. Let $\ell \in \mathbb{N}$ with $\ell \geqslant 4$. Builder can force one of the following structures independent of Painter's choices:

(i) a red $P_{3}$ in at most $5 \ell / 4-1$ rounds.

(ii) a blue $P_{\ell+1}$ in at most $5 \ell / 4-1$ rounds.

(iii) a blue $P_{t}$ with one endpoint incident to a red edge in at most $5 t / 4-1$ rounds for some $\ell-3 \leqslant t \leqslant \ell$.

Proof. Throughout the proof, we assume for clarity that Painter will always avoid (i) and (ii) if possible. By Lemma 19 (taking $q=\ell+1$ ) we may assume that Builder has constructed a blue $P_{t}$, say $v_{1} \ldots v_{t}$, which satisfies

(*) $v_{1} \ldots v_{t}$ has one endpoint incident to a red edge $v_{1} u$, and Builder constructed $v_{1} \ldots v_{t}$ in at most $5 t / 4-1$ rounds. Moreover, $4 \leqslant t \leqslant \ell$.

Note that $t \leqslant 5 t / 4-1$ since $t \geqslant 4$.

If $t \geqslant \ell-3$, then we have achieved (iii). Hence, we may assume that $4 \leqslant t<\ell-3$. Without loss of generality, let $v_{1} u$ be a red edge as in $(*)$. Builder will extend $v_{1} \ldots v_{t}$ as follows. We apply Lemma 19 with $q=\ell-t+1 \geqslant 5$ on a set of new vertices. We split into cases depending on Painter's choice.

Case 1: Builder obtains a red $P_{3}$ in at most $\ell-t$ rounds, as in Lemma 19(i). 
In this case, Builder has spent at most $5 t / 4-1+\ell-t \leqslant 5 \ell / 4-2$ rounds in total since $t \leqslant \ell-4$, and so we have achieved (i).

Case 2: Builder obtains a blue path $w_{1} \ldots w_{\ell-t+1}$ in $\ell-t$ rounds, as in Lemma 19(ii).

In this case, Builder has again spent at most $5 \ell / 4-2$ rounds in total. Builder now chooses the edge $w_{1} v_{1}$. If Painter colours it red, then $w_{1} v_{1} u$ is a red $P_{3}$ and we have achieved (i). If Painter colours it blue, then $w_{\ell-t+1} \ldots w_{1} v_{1} \ldots v_{t}$ is a blue $P_{\ell+1}$ and we have achieved (ii).

Case 3: Builder obtains a blue path $w_{1} \ldots w_{t^{\prime}}$ and a red edge $w_{1} x$ in at most $t^{\prime}$ rounds for some $4 \leqslant t^{\prime} \leqslant \ell-t$, as in Lemma 19(iii).

In this case, Builder has spent at most

$$
\frac{5 t}{4}-1+t^{\prime}=\frac{5 t}{4}+\frac{5 t^{\prime}}{4}-\frac{t^{\prime}}{4}-1 \leqslant \frac{5\left(t+t^{\prime}\right)}{4}-2 \leqslant \frac{5 \ell}{4}-2
$$

rounds in total. Builder now chooses the edge $v_{t} w_{1}$. If Painter colours it red, then $v_{t} w_{1} x$ is a red $P_{3}$ and we have achieved (i). If Painter colours it blue, then $v_{1} \ldots v_{t} w_{1} \ldots w_{t^{\prime}}$ is a blue $P_{t+t^{\prime}}$ with $v_{1}$ incident to the red edge $v_{1} u$. Moreover, this $P_{t+t^{\prime}}$ satisfies $(*)$ with $t+t^{\prime}>t$. Hence by iterating the argument above, the result follows.

Theorem 21. For all $\ell \geqslant 2, \tilde{r}\left(P_{3}, P_{\ell+1}\right)=\lceil 5 \ell / 4\rceil$.

Proof. Theorem 4 implies that $\tilde{r}\left(P_{3}, P_{\ell+1}\right) \geqslant\lceil 5 \ell / 4\rceil$. It therefore suffices to prove that Builder can win the $\tilde{r}\left(P_{3}, P_{\ell+1}\right)$-game within $\lceil 5 \ell / 4\rceil$ rounds. First note that $\tilde{r}\left(P_{3}, P_{3}\right)=$ 3 and $\tilde{r}\left(P_{3}, P_{4}\right)=4$, as shown by Grytczuk, Kierstead and Prałat [8] and Prałat [13] respectively, so we may assume $\ell \geqslant 4$. Applying Lemma 20, either Builder obtains a blue path $v_{1} \ldots v_{t+1}$ and a red edge $v_{1} u$ in at most $5(t+1) / 4-1$ rounds for some $\ell-3 \leqslant t+1 \leqslant \ell$ or we are done. Write

$$
r(t)=\left\lceil\frac{5 \ell}{4}\right\rceil-\left(\left\lfloor\frac{5(t+1)}{4}\right\rfloor-1\right)=\left\lceil\frac{\ell}{4}\right\rceil-\left\lfloor\frac{t+1}{4}\right\rfloor+(\ell-t),
$$

and note that Builder has at least $r(t)$ rounds left to construct either a red $P_{3}$ or a blue $P_{\ell+1}$. We now split into cases depending on the precise value of $t$.

Case 1: $t=\ell-1$, so that $r(t)=1$.

Builder chooses the edge $v_{0} v_{1}$, where $v_{0}$ is a new vertex. If Painter colours it red, then $v_{0} v_{1} u$ is a red $P_{3}$ and we are done. Otherwise, $v_{0} v_{1} \ldots v_{\ell}$ is a blue $P_{\ell+1}$ and we are done.

Case 2: $t=\ell-2$, so that $r(t) \geqslant 3$.

Builder chooses the edge $v_{\ell-1} x$, where $x$ is a new vertex. If Painter colours it blue, then we are in Case 1 with an extra round to spare. If Painter colours it red, Builder chooses the edges $v_{\ell-1} w$ and $w x$, where $w$ is a new vertex. If Painter colours either edge red then $x v_{\ell-1} w$ or $w x v_{\ell-1}$ respectively is a red $P_{3}$ and we are done. Otherwise, $v_{1} \ldots v_{\ell-1} w x$ is a blue $P_{\ell+1}$ and we are done.

Case 3: $t=\ell-3$, so that $r(t) \geqslant 4$. 
Builder chooses the edge $v_{\ell-2} x$, where $x$ is a new vertex. If Painter colours it blue, then we are in Case 2. If Painter colours it red, Builder chooses the edges $v_{\ell-2} w, w x$ and $x y$, where $w$ and $y$ are new vertices. If Painter colours any of these edges red then $x v_{\ell-2} w$, $w x v_{\ell-2}$ or $v_{\ell-2} x y$ respectively is a red $P_{3}$ and we are done. Otherwise, $v_{1} \ldots v_{\ell-2} w x y$ is a blue $P_{\ell+1}$ and we are done.

Case 4: $t=\ell-4$, so that $r(t) \geqslant 5$.

Builder chooses the edge $v_{\ell-3} x$, where $x$ is a new vertex. If Painter colours it blue, then we are in Case 3. If Painter colours it red, Builder chooses the edges $v_{0} v_{1}, v_{\ell-3} w$, $w x$ and $x y$, where $v_{0}, w$ and $y$ are new vertices. If Painter colours any of these edges red then $v_{0} v_{1} u, x v_{\ell-3} w, w x v_{\ell-3}$ or $v_{\ell-3} x y$ respectively is a red $P_{3}$ and we are done. Otherwise, $v_{0} v_{1} \ldots v_{\ell-3} w x y$ is a blue $P_{\ell+1}$ and we are done.

\section{Determining $\tilde{r}\left(P_{3}, C_{\ell}\right)$ for $\ell \geqslant 3$}

Our aim is to determine $\tilde{r}\left(P_{3}, C_{\ell}\right)$ for all $\ell \geqslant 3$, so proving Theorem 5 . As a warmup, we first determine $\tilde{r}\left(P_{3}, C_{3}\right)$ and $\tilde{r}\left(P_{3}, C_{4}\right)$. Note that Theorem 4 implies that $\tilde{r}\left(P_{3}, C_{3}\right) \geqslant 5 \ell / 4$ for all $\ell \geqslant 3$, but this lower bound is too weak when $\ell \leqslant 4$. Instead, we consider the $\left\{C_{\ell}\right\}$-blocking strategy for Painter in an $\tilde{r}\left(C_{\ell}, P_{3}\right)$-game.

Proposition 22. For all $\ell \geqslant 3$, we have $\tilde{r}\left(P_{3}, C_{\ell}\right) \geqslant \ell+2$.

Proof. We consider the $\left\{C_{\ell}\right\}$-blocking strategy for Painter in the $\tilde{r}\left(C_{\ell}, P_{3}\right)$-game. Let $R$ be an edge-minimal $\left\{C_{\ell}\right\}$-scaffolding for $P_{3}$. Then $R$ must contain two distinct $P_{\ell}$ 's, so $e(R) \geqslant \ell$. The result therefore follows from Proposition 13.

The upper bounds are both relatively straightforward.

Proposition 23. We have $\tilde{r}\left(P_{3}, C_{3}\right)=5$ and $\tilde{r}\left(P_{3}, C_{4}\right)=6$.

Proof. By Proposition 22, we have $\tilde{r}\left(P_{3}, C_{3}\right) \geqslant 5$ and $\tilde{r}\left(P_{3}, C_{4}\right) \geqslant 6$. It is easy to show that $r\left(P_{3}, C_{4}\right)=4$ (see e.g. Radziszowski [15]), so we also have $\tilde{r}\left(P_{3}, C_{4}\right) \leqslant\left(\begin{array}{l}4 \\ 2\end{array}\right)=6$ as Builder may simply choose the edges of a $K_{4}$. It therefore suffices to prove that Builder can win the $\tilde{r}\left(P_{3}, C_{3}\right)$-game in 5 rounds.

Take new vertices $u, v, w, x, y$ and $z$. Builder first chooses the edges $u v, u w$ and $u x$. If Painter colours more than one of these edges red, then we have obtained a red $P_{3}$ and we are done.

Suppose Painter colours $u v, u w$ and $u x$ blue. Then Builder chooses the edges $v w$ and $w x$. If Painter colours either edge blue, then vwuv or wxuw respectively is a blue $C_{3}$ and we are done. If Painter colours both edges red, then $v w x$ is a red $P_{3}$ and we are done.

Finally, suppose Painter colours (without loss of generality) $u v$ red, but $u w$ and $u x$ blue. Then Builder chooses the edge $x y$. If Painter colours $x y$ red, Builder chooses the edge $w x$, yielding either a red $P_{3}$ (namely $w x y$ ), or a blue $C_{3}, w x u w$, and we are done. If Painter colours $x y$ blue, Builder chooses the edge $y u$, yielding either a red $P_{3}$ (namely yuv) or a blue $C_{3}$ (namely uxyu), and we are done. 
We now determine $\tilde{r}\left(P_{3}, C_{\ell}\right)$ for $\ell \geqslant 5$. As in Section 4, Builder's strategy will be to build up a long blue path using Lemma 20. Builder will then carefully close this path into a blue $C_{\ell}$.

Theorem 24. For all $\ell \geqslant 5, \tilde{r}\left(P_{3}, C_{\ell}\right)=\lceil 5 \ell / 4\rceil$.

Proof. Theorem 4 implies that $\tilde{r}\left(P_{3}, C_{\ell}\right) \geqslant\lceil 5 \ell / 4\rceil$. It therefore suffices to prove that Builder can win the $\tilde{r}\left(P_{3}, C_{\ell}\right)$-game within $\lceil 5 \ell / 4\rceil$ rounds. By Lemma 20, Builder can force one of the following structures independent of Painter's choices:

(i) a red $P_{3}$ in at most $5(\ell-1) / 4-1$ rounds.

(ii) a blue $P_{\ell}$ in at most $5(\ell-1) / 4-1$ rounds.

(iii) a blue $P_{t}$ with one endpoint incident to a red edge in at most $5 t / 4-1$ rounds for some $\ell-4 \leqslant t \leqslant \ell-1$.

If Painter chooses (i), then we are done. Suppose Painter chooses (ii), so that Builder has at least

$$
\left\lceil\frac{5 \ell}{4}\right\rceil-\left(\frac{5(\ell-1)}{4}-1\right)=\left\lceil\frac{5 \ell}{4}\right\rceil-\frac{5 \ell}{4}+\frac{9}{4}>2
$$

rounds to construct a red $P_{3}$ or a blue $C_{\ell}$, and let $v_{1} \ldots v_{\ell}$ be the corresponding blue path. Then Builder chooses the edges $v_{\ell} v_{1}, v_{1} v_{3}$ and $v_{\ell} v_{2}$. If Painter colours $v_{\ell} v_{1}$ blue then $v_{1} \ldots v_{\ell} v_{1}$ is a blue $C_{\ell}$ and we are done. If Painter colours $v_{\ell} v_{1}$ red and $v_{1} v_{3}$ or $v_{\ell} v_{2}$ red, then $v_{\ell} v_{1} v_{3}$ or $v_{1} v_{\ell} v_{2}$ respectively is a red $P_{3}$ and we are done. Finally, if Painter colours both $v_{1} v_{3}$ and $v_{\ell} v_{2}$ blue, then $v_{1} v_{3} v_{4} \ldots v_{\ell} v_{2} v_{1}$ is a blue $C_{\ell}$ and we are done.

Finally, suppose Painter chooses (iii). Let $v_{1} \ldots v_{t}$ be the corresponding blue path and let $v_{1} u$ be a red edge. Write

$$
r(t)=\left\lceil\frac{5 \ell}{4}\right\rceil-\left(\left\lfloor\frac{5 t}{4}\right\rfloor-1\right)=\left\lceil\frac{\ell}{4}\right\rceil-\left\lfloor\frac{t}{4}\right\rfloor+\ell-t+1
$$

so that Builder has at least $r(t)$ rounds left to construct either a red $P_{3}$ or a blue $C_{\ell}$. We split into cases depending on the precise value of $t$.

Case 1: $t=\ell-1$, so that $r(t) \geqslant 3$.

Builder first chooses the edge $v_{\ell-1} w$, where $w$ is a new vertex. If Painter colours $v_{\ell-1} w$ blue, then Builder chooses the edge $w v_{1}$. If Painter colours $w v_{1}$ red then $w v_{1} u$ is a red $P_{3}$, and if Painter colours $w v_{1}$ blue then $v_{1} v_{2} \ldots v_{\ell-1} w v_{1}$ is a blue $C_{\ell}$. Now suppose Painter colours $v_{\ell-1} w$ red instead. Then Builder chooses the edges $v_{\ell-1} x$ and $x v_{1}$, where $x$ is a new vertex. If Painter colours either edge red, then $w v_{\ell-1} x$ or $x v_{1} u$ respectively is a red $P_{3}$ and we are done. Otherwise, $v_{1} \ldots v_{\ell-1} x v_{1}$ is a blue $C_{\ell}$ and we are done.

Case 2: $t=\ell-2$, so that $r(t) \geqslant 4$.

Builder first chooses the edge $v_{\ell-2} w$, where $w$ is a new vertex. If Painter colours $v_{\ell-2} w$ blue then we are in Case 1, so suppose Painter colours $v_{\ell-2} w$ red. Builder then chooses the edges $v_{\ell-2} x, x w$ and $w v_{1}$, where $x$ is a new vertex. If Painter colours any of these edges 
red, then $w v_{\ell-2} x, x w v_{\ell-2}$ or $v_{\ell-2} w v_{1}$ respectively is a red $P_{3}$ and we are done. Otherwise, $v_{1} v_{2} \ldots v_{\ell-2} x w v_{1}$ is a blue $C_{\ell}$ and we are done.

Case 3: $t=\ell-3$, so that $r(t) \geqslant 5$.

Builder first chooses the edge $v_{\ell-3} w$, where $w$ is a new vertex. If Painter colours $v_{\ell-3} w$ blue then we are in Case 2, so suppose Painter colours $v_{\ell-3} w$ red. Builder then chooses the edges $v_{\ell-3} x, x w, w y$ and $y v_{1}$, where $x$ and $y$ are new vertices. If Painter colours any of these edges red, then $w v_{\ell-3} x, x w v_{\ell-3}, v_{\ell-3} w y$ or $y v_{1} u$ respectively is a red $P_{3}$ and we are done. Otherwise, $v_{1} v_{2} \ldots v_{\ell-3} x w y v_{1}$ is a blue $C_{\ell}$ and we are done.

Case 4: $t=\ell-4$, so that $r(t) \geqslant 6$.

Builder first chooses two edges $w x$ and $x y$, where $w, x$ and $y$ are new vertices. If Painter colours both edges red, wxy is a red $P_{3}$ and we are done. Now suppose that Painter colours one edge blue and one red, say $w x$ red and $x y$ blue. Then Builder chooses the edges $v_{\ell-4} w, w z, z x$ and $y v_{1}$, where $z$ is a new vertex. If Painter colours any of these edges red, then $v_{\ell-4} w x, x w z, z x w$ or $y v_{1} u$ respectively is a red $P_{3}$ and we are done. Otherwise, $v_{1} v_{2} \ldots v_{\ell-4} w z x y v_{1}$ is a blue $C_{\ell}$ and we are done.

We may therefore assume that Painter colours both $w x$ and $x y$ blue. Builder now chooses the edge $v_{\ell-4} w$. If Painter colours $v_{\ell-4} w$ blue, we are in Case 1 (taking our path to be $v_{1} v_{2} \ldots v_{\ell-4} w x y$ ), so suppose Painter colours $v_{\ell-4} w$ red. Then Builder chooses the edges $v_{\ell-4} z, z w$ and $y v_{1}$, where $z$ is a new vertex. If Painter colours any of these edges red, then $w v_{\ell-4} z, z w v_{\ell-4}$ or $y v_{1} u$ respectively is a red $P_{3}$ and we are done. Otherwise, $v_{1} v_{2} \ldots v_{\ell-4} z w x y v_{1}$ is a blue $C_{\ell}$ and we are done.

\section{Bounding $\tilde{r}\left(C_{4}, P_{\ell+1}\right)$ for $\ell \geqslant 3$}

Our aim is to prove Theorem 9 , i.e. to bound $\tilde{r}\left(C_{4}, P_{\ell+1}\right)$ for all $\ell \geqslant 3$. First we prove that $\tilde{r}\left(C_{4}, P_{4}\right)=8$.

Proposition 25. $\tilde{r}\left(C_{4}, P_{4}\right)=8$.

Proof. First, we consider the $\left\{C_{4}\right\}$-blocking strategy for Painter in the $\tilde{r}\left(C_{4}, P_{4}\right)$-game. Let $R$ be an edge-minimal $\left\{C_{4}\right\}$-scaffolding for $P_{4}$. Then $R$ must contain three distinct $P_{4}$ 's, so $e(R) \geqslant 5$ as $R$ is $C_{4}$-free. Proposition 13 implies that $\tilde{r}\left(C_{4}, P_{4}\right) \geqslant 8$.

It therefore suffices to prove that Builder can win the $\tilde{r}\left(C_{4}, P_{4}\right)$-game within 8 rounds. Builder first chooses the edges $u v_{1}, \ldots, u v_{4}$ for distinct vertices $u, v_{1}, \ldots, v_{4}$. Without loss of generality we may assume that there exists an integer $j$ such that Painter colours the edges $u v_{i}$ blue if $i \leqslant j$, and red otherwise.

Suppose $j \geqslant 2$. Then Builder chooses four edges $v_{1} w, v_{2} w, v_{1} w^{\prime}$ and $v_{2} w^{\prime}$, where $w$ and $w^{\prime}$ are new vertices. If Painter colours all edges red, then $v_{1} w v_{2} w^{\prime} v_{1}$ is a red $C_{4}$. If Painter colours one of the edges blue say $v_{2} w$, then $v_{1} u v_{2} w$ is a blue $P_{4}$.

Suppose $j \leqslant 1$. Then Builder chooses edges $v_{1} v_{2}$ and $v_{1} v_{3}$. If Painter colours both edges red, then $u v_{2} v_{1} v_{3} u$ is a red $C_{4}$. Suppose that Painter colours both edges blue. Builder then chooses the edges $v_{2} v_{4}$ and $v_{3} v_{4}$. If Painter colours both $v_{2} v_{4}$ and $v_{3} v_{4}$ red, then $u v_{2} v_{4} v_{3} u$ is a red $C_{4}$. Otherwise, $v_{3} v_{1} v_{2} v_{4}$ or $v_{2} v_{1} v_{3} v_{4}$ is a blue $P_{4}$. Therefore we may 
assume that $v_{1} v_{2}$ is blue and $v_{1} v_{3}$ is red. Further suppose that $j=1$ and so $u v_{1}$ is blue. Then Builder chooses the edges $v_{2} v_{3}$ and $v_{2} v_{4}$. If Painter colours one of them blue, then $u v_{1} v_{2} v_{3}$ or $u v_{1} v_{2} v_{4}$ is a blue $P_{4}$. Otherwise $u v_{3} v_{2} v_{4} u$ is a red $C_{4}$. Finally, suppose that $j=0$. Builder chooses the edges $v_{2} v_{3}$ and $v_{3} v_{4}$. If Painter colours one of them red, then $u v_{1} v_{3} v_{2} u$ or $u v_{1} v_{3} v_{4} u$ is a red $C_{4}$. Otherwise $v_{1} v_{2} v_{3} v_{4}$ is a blue $P_{4}$.

We now prove Theorem 9 .

Proof of Theorem 9. The lower bound follows from Lemma 14 and $\tilde{r}\left(C_{4}, P_{4}\right)=8$ by Proposition 25. To prove the theorem, it is enough to show that $\tilde{r}\left(C_{4}, P_{\ell+1}\right) \leqslant 4 \ell-4$ for all $\ell \geqslant 3$. We proceed by induction on $\ell$. By Proposition 25, this is true for $\ell=3$. Suppose instead that $\ell \geqslant 4$ and Builder first spends at most $4 \ell-8$ rounds forcing Painter to construct a red $C_{4}$ or a blue $P_{\ell}=v_{1} \ldots v_{\ell}$. (This is possible by the induction hypothesis.) We may assume that the latter holds or else we are done. Then Builder chooses four edges $v_{1} x, v_{\ell} x, v_{1} y$ and $v_{\ell} y$, where $x$ and $y$ are new vertices. If Painter colours all edges red, then $v_{1} x v_{\ell} y v_{1}$ is a red $C_{4}$. If Painter colours one of the edges blue, say $v_{\ell} x$, then $v_{1} \ldots v_{\ell} x$ is a blue $P_{\ell+1}$. In total, Builder has chosen at most $4 \ell-4$ edges and the proposition follows.

\section{Acknowledgements}

We would like to thank the referee for pointing out an error in the early manuscript.

\section{References}

[1] J. Beck. Achievement games and the probabilistic method. Combinatorics, Paul Erdös is Eighty, Bolyai Society of Mathematical Studies, 1:51-78, 1993.

[2] J. Beck. On size Ramsey number of paths, trees and cycles I. Journal of Graph Theory, 7:115-130, 1983.

[3] D. Conlon. On-line Ramsey numbers. SIAM Journal on Discrete Mathematics, 23(4):1954-1963, 2009.

[4] A. Dudek and P. Prałat. An alternative proof of the linearity of the size-Ramsey number of paths. Combinatorics, Probability and Computing, in press.

[5] P. Erdős, R. J. Faudree, C. C. Rousseau and R. H. Schelp. The size Ramsey number. Periodica Mathematica Hungarica, 9:145-161, 1978.

[6] E. Friedgut, Y. Kohayakawa, V. Rödl, A. Ruciński and P. Tetali. Ramsey games against a one-armed bandit. Combinatorics, Probability and Computing, 12:515-545, 2003.

[7] J. Grytczuk, M. Hałuszczak and H. Kierstead. On-line Ramsey theory. Electronic Journal of Combinatorics, 11(1):\#R57, 2004.

[8] J. Grytczuk, H. Kierstead and P. Prałat. On-line Ramsey numbers for paths and stars. Discrete Mathematics and Theoretical Computer Science, 10(3):63-74, 2008. 
[9] H. Kierstead and G. Konjevod. Coloring number and on-line Ramsey theory for graphs and hypergraphs. Combinatorica, 29:49-64, 2009.

[10] A. Kurek and A. Ruciński. Two variants of the size Ramsey number. Discussiones Mathematicae Graph Theory, 25:141-149, 2005.

[11] M. Marciniszyn, R. Spöhel, and A. Steger. Upper bounds for online Ramsey games in random graphs. Combinatorics, Probability and Computing, 18:259-270, 2009.

[12] M. Marciniszyn, R. Spöhel, and A. Steger. Online Ramsey games in random graphs. Combinatorics, Probability and Computing, 18:271-300, 2009.

[13] P. Prałat. A note on off-diagonal small on-line Ramsey numbers for paths. Ars Combinatoria, 107:295-306, 2012.

[14] P. Prałat. A note on small on-line Ramsey numbers for paths and their generalisations. Australasian Journal of Combinatorics, 40:27-36, 2008.

[15] S. Radziszowski. Small Ramsey numbers. Electronic Journal of Combinatorics, DS1, 2014.

[16] F. Ramsey. On a problem of formal logic. Proceedings of the London Mathematical Society, 30:264-286, 1930.

\section{A Bounding $\tilde{\boldsymbol{r}}\left(\boldsymbol{P}_{4}, \boldsymbol{P}_{\ell+1}\right)$ for $\ell \geqslant 3$}

Here, we prove Theorem 6. Lemma 18 implies that $\tilde{r}\left(P_{4}, P_{\ell+1}\right) \geqslant(7 \ell+2) / 5$ for $\ell \geqslant 3$. It therefore suffices to bound $\tilde{r}\left(P_{4}, P_{\ell+1}\right)$ above, which we do in Theorem 44 . In the following discussion we take on the role of Builder, and we will assume for clarity that Painter will not voluntarily lose the game by creating a red $P_{4}$.

We will employ the following strategy to construct a blue $P_{\ell+1}$. We will obtain two (initially trivial) vertex-disjoint blue paths $Q$ and $R$, repeatedly extend them, and then join them together to form a blue $P_{\ell+1}$ when they are sufficiently long. Here $Q$ is distinct from $R$ in that we require one of $Q$ 's endpoints to be incident to a red edge $b c$ disjoint from $V(R)$. Some of our methods for extending a blue path require this property, and others destroy it. Thus at each stage we will extend either $Q$ or $R$ depending on which of our extension methods Painter allows us to use.

We will use the following lemma to join $Q$ and $R$ together (and sometimes to extend $Q$ ).

Lemma 26. Let $Q$ be a (possibly trivial) blue path with endpoints $a$ and $b$, where $b$ is incident to a red edge bc. Let $R$ be a (possibly trivial) blue path vertex-disjoint from $V(Q) \cup\{c\}$. Then Builder can force Painter to construct one of the following while uncovering at most 2 edges:

(i) a blue path $Q^{\prime}$ of length $e(Q)+e(R)+1$ with one endpoint incident to a red edge.

(ii) a red $P_{4}$.

Proof. First suppose that $R$ is non-trivial, and let $x$ and $y$ be the endpoints of $R$. Moreover, suppose that either $a=c$ or $Q$ is trivial, so that both endpoints of $Q$ are incident 
to $b c$. Builder chooses the edges $b x$ and $c y$. If Painter colours both edges red, then $x b c y$ is a red $P_{4}$. Hence, without loss of generality, we may assume that Painter colours $b x$ blue. Then $Q^{\prime}:=a Q b x R y$ is a blue path of length $e(Q)+e(R)+1$, where $a$ is incident to the red edge $b c$.

Now suppose that $Q$ is non-trivial and $a \neq c$. Builder chooses the edge $a x$. If Painter colours ax blue, then bQaxRy is a blue path of length $e(Q)+e(R)+1$ with endpoint $b$ incident to the red edge $b c$. So we may assume that Painter colours ax red. Builder then chooses the edge $b x$. If Painter colours $b x$ red, then $c b x a$ is a red $P_{4}$. Otherwise $Q^{\prime}:=a Q b x R y$ is a blue path of length $e(Q)+e(R)+1$ where $a$ is incident to the red edge $a x$.

Finally, suppose $R$ is trivial with endpoint $x$. Let $y$ be a new vertex. Then the argument above implies the lemma on replacing $x R y$ with $x$ throughout.

The arguments that follow are by necessity somewhat technical. The reader may therefore find the following intuition useful.

(i) For every seven edges we uncover, we will extend either $Q$ or $R$ by five blue edges.

(ii) When we join $Q$ and $R, e(Q)+e(R)+1$ should not be too much greater than $\ell$.

It is clear that following the above principles will yield a bound of the form $\tilde{r}\left(P_{4}, P_{\ell+1}\right) \leqslant$ $7 \ell / 5+C$ for some constant $C$. We will violate (i) in the first and last phases of Builder's strategy, but this introduces only constant overhead.

Before we can apply Lemma 26 to join $Q$ and $R$ and obtain a blue $P_{\ell+1}$, we must extend them until $e(Q)+e(R)+1 \geqslant \ell$. Each time we extend $Q$ and $R$, we require two independent edges of the same colour. (Naturally, we can obtain these by choosing three independent edges.) If these edges are blue, we may extend $Q$ efficiently using Lemma 30 (see Section A.1). If they are red, we may extend either $Q$ or $R$ efficiently using Lemma 39 (see Section A.2). Note that the latter case is significantly harder. We then apply Lemmas 30 and 39 repeatedly to prove Theorem 44 (see Section A.3).

In our figures throughout the section, we shall represent blue edges with solid lines and red edges with dotted lines.

\section{A.1 Extending $Q$ using two independent blue edges $e$ and $f$.}

Throughout this subsection, $e$ and $f$ will be two independent blue edges vertex-disjoint from $Q$ and $R$. We will prove that we can use these two edges to efficiently extend $Q$ - see Lemma 30. We first define a special type of path which will be important to the extension process.

Definition 27. We say that a path $x y S z$ is of type $A$ if $x y$ is a red edge and $S$ is a non-trivial blue path with endpoints $y$ and $z$.

Note that the above definition requires $x \notin V(S)$. For the remainder of the section, if we refer to a path $x y S z$ of type A, we shall take it as read that $x, y, z$ and $S$ are as in Definition 27. 
We now sketch the proof of Lemma 30. By greedily extending the blue edge $e$ into a path, Builder can obtain either a long blue path or a path of type A (see Lemma 28). If Builder obtains a long blue path $P$, then we can simply join $P$ and $Q$ together using Lemma 26. Suppose instead Builder obtains a path $x y S z$ of type A. Then we use Lemma 29 to efficiently join $S$ and $Q$ together. In either case, the resulting blue path $Q^{\prime}$ also has an endpoint incident to a red edge, so $Q^{\prime}$ retains the defining property of $Q$.

We first prove that Builder can obtain either a long blue path or a path of type A by greedily extending $e$.

Lemma 28. Let $m \in \mathbb{N}$ and let $e$ be a blue edge. Then Builder can force Painter to construct one of the following:

(i) a path $x y S z$ of type $A$ with $e(S)=t$ while uncovering $t$ edges for some $1 \leqslant t<m$.

(ii) a blue path of length $m$ while uncovering $m-1$ edges.

Proof. Let $S_{1}$ be the blue path formed by $e$. Builder proceeds to extend $S_{1}$ greedily until either Builder has constructed a blue path of length $m$ or Painter has coloured an edge red.

Indeed, suppose $S_{i}$ is a blue path of length $i$ for some $1 \leqslant i \leqslant m-1$ with endpoints $y$ and $z$, and that Builder has uncovered $i-1$ edges in forming $S_{i}$ from $S_{1}$. Then Builder chooses the edge $x y$, where $x$ is a new vertex. If Painter colours $x y$ red then $x y S_{i} z$ is a path of type A with $e\left(S_{i}\right)=i$, where $1 \leqslant i<m$. Moreover, Builder has uncovered $i$ edges in constructing it, and so we have achieved (i). If instead Painter colours $x y$ blue, then $S_{i+1}:=x y S_{i} z$ is a blue path of length $i+1$ and Builder has uncovered $i$ edges in constructing it.

By repeating this process, Builder must either obtain a path of type A as in (i) or a blue path $S_{m}$ of length $m$ as in (ii).

We now prove that Builder can use a path of type A to efficiently extend $Q$. Recall that we were given two independent blue edges, $e$ and $f$, and that we have already used $e$ to construct a path of type A.

Lemma 29. Suppose $Q$ is a non-trivial blue path with endpoints $a$ and $b$, where $b$ is incident to a red edge bc.Suppose $x y S z$ is a path of type $A$ which is vertex-disjoint from $V(Q) \cup\{c\}$. Further suppose that $f=v w$ is a blue edge vertex-disjoint from $V(Q) \cup$ $V(x y S z) \cup\{c\}$. Then Builder can force Painter to construct one of the following:

(i) a blue path $Q^{\prime}$ of length $e(Q)+e(S)+2$ with one endpoint $b^{\prime}$ incident to a red edge $b^{\prime} c^{\prime}$ while uncovering 2 edges. Moreover, $f$ is vertex-disjoint from $V\left(Q^{\prime}\right) \cup\left\{c^{\prime}\right\}$.

(ii) a blue path $Q^{\prime}$ of length $e(Q)+e(S)+4$ with one endpoint incident to a red edge $b^{\prime} c^{\prime}$ while uncovering 4 edges. (Note that $f$ need not be vertex-disjoint from $V\left(Q^{\prime}\right) \cup\left\{c^{\prime}\right\}$.)

(iii) a red $P_{4}$ while uncovering at most 4 edges. 

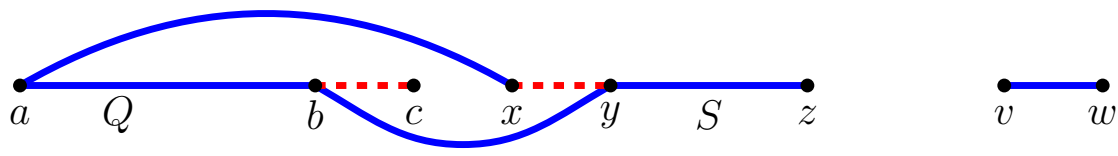

Figure 1: Extending $Q$ using a path of type A as in Lemma 29(i).

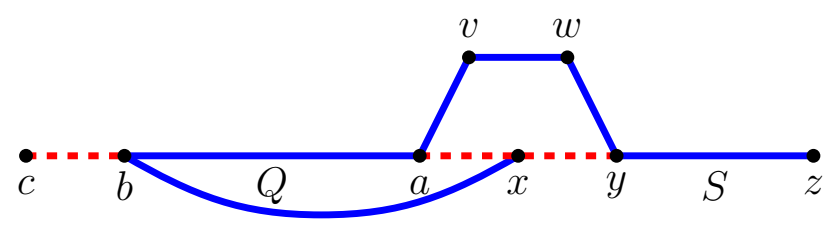

Figure 2: Extending $Q$ using a path of type $\mathrm{A}$ and an blue independent edge $v w$ as in Lemma 29(ii).

Proof. Builder chooses the edge ax. First suppose Painter colours ax blue. Builder then chooses the edge by. If Painter colours the edge by red, then cbyx is a red $P_{3}$ and we have achieved (iii). Suppose not. Then $Q^{\prime}:=x a Q b y S z$ (see Figure 1) is a blue path of length $e(Q)+e(S)+2$, where $x$ is incident to the red edge $x y$, and we have achieved (i).

Now suppose Painter instead colours ax red. Builder then chooses the edges av, wy and $x b$. If Painter colours any of these edges red, then yxav, wyxa or yxbc respectively is a red $P_{4}$ and we have achieved (iii). Suppose not. Then $Q^{\prime}:=x b$ Qavwy $S z$ (see Figure 2) is a blue path of length $e(Q)+e(S)+4$, where $x$ is incident to the red edge $x y$, and we have achieved (ii).

We now consolidate Lemmas 28 and 29 into a single lemma which says that given two independent blue edges, Builder can efficiently extend $Q$. In applying Lemma 30, we will take $m$ to be $\ell-e(Q)-e(R)-1$. Thus if we can extend $Q$ by at least $m$ edges, then we can join $Q$ and $R$ to obtain a blue $P_{\ell+1}$ immediately afterwards.

Lemma 30. Let $m \in \mathbb{N}$. Suppose $Q$ is a non-trivial blue path with endpoints a and $b$, where $b$ is incident to a red edge bc. Suppose $e$ and $f$ are two independent blue edges which are vertex-disjoint from $V(Q) \cup\{c\}$. Then Builder can force Painter to construct one of the following:

(i) a blue path $Q^{\prime}$ with $e\left(Q^{\prime}\right)=e(Q)+\ell^{\prime}$ for some $3 \leqslant \ell^{\prime} \leqslant m+3$ such that $Q^{\prime}$ has an endpoint $b^{\prime}$ incident to a red edge $b^{\prime} c^{\prime}$. A total of $\ell^{\prime}$ edges are uncovered in the process. Moreover, if $\ell^{\prime}<5 \leqslant m$, then $f$ is vertex-disjoint from $V\left(Q^{\prime}\right) \cup\left\{c^{\prime}\right\}$.

(ii) a red $P_{4}$ while uncovering at most $m+3$ edges.

Proof. We apply Lemma 28 to $e$ and $m$, and split into cases depending on Painter's choice.

Case 1: As in Lemma 28(i), we obtain a path $x y S z$ of type A with $e(S)=t$ for some $1 \leqslant t<m$ which is vertex-disjoint from $V(f) \cup V(Q) \cup\{c\}$, while uncovering $t$ edges.

We apply Lemma 29 to $Q, x y S z$ and $f$. First suppose that as in Lemma 29(i), we obtain a blue path $Q^{\prime}$ of length $e(Q)+t+2$ with one endpoint incident to a red edge while 
preserving $f^{\prime}$ 's independence. In total we have uncovered $t+2$ edges. Hence $Q^{\prime}$ satisfies (i) on setting $\ell^{\prime}=t+2$.

Now suppose that as in Lemma 29(ii), we obtain a blue path $Q^{\prime}$ of length $e(Q)+t+4$ with one endpoint incident to a red edge. We have uncovered $t+4$ edges in total. Hence setting $\ell^{\prime}=t+4$, we have achieved (i) with $\ell^{\prime} \geqslant 5$.

Finally, suppose that as in Lemma 29(iii) we obtain a red $P_{4}$. Then we have uncovered at most $t+4 \leqslant m+3$ edges in total and so we have achieved (ii).

Case 2: As in Lemma 28(ii), we obtain a blue path $S$ of length $m$ which is vertex-disjoint from $V(Q) \cup\{c\}$ while uncovering $m-1$ edges.

We apply Lemma 26 to $Q$ and $S$ to construct either a blue path $Q^{\prime}$ of length $e(Q)+m+1$ with one endpoint incident to a red edge or a red $P_{4}$ while uncovering at most 2 additional edges. We have uncovered at most $m+1$ edges in total. Hence in the former case we have achieved (i), and in the latter case we have achieved (ii).

\section{A.2 Extending $Q$ and $R$ using two red edges $e$ and $f$.}

In this subsection, our aim is to extend $Q$ or $R$ efficiently when given two independent red edges $e$ and $f$ - see Lemma 39. As in Section A.1, it will be convenient to define some special paths that we will use in the extension process. These paths can be viewed as analogues of paths of type A.

Definition 31. A path $v w x y z$ is of type $B$ if $v w$ and $y z$ are red edges, and $w x$ and $x y$ are blue edges.

Definition 32. A path $T_{1} \ldots T_{k}$ is of type $C$ if the following statements hold:

(C1) $k$ is odd and $k \geqslant 3$.

(C2) $T_{1}$ is either a blue edge or a path of the form $x_{1} y_{1} z_{1}$, where $z_{1} \in V\left(T_{2}\right)$ and $y_{1} z_{1}$ is red (and $x_{1} y_{1}$ may be red or blue).

(C3) $T_{k}$ is either a blue edge or a path of the form $x_{k} y_{k} z_{k}$, where $x_{k} \in V\left(T_{k-1}\right)$ and $x_{k} y_{k}$ is red (and $y_{k} z_{k}$ may be red or blue).

(C4) $T_{2}, T_{4}, \ldots, T_{k-1}$ are blue paths. Exactly one of these paths has length 1 and the rest have length 2 .

(C5) $T_{3}, T_{5}, \ldots, T_{k-2}$ are all red $P_{3}$ 's.

We say $T_{1} \ldots T_{k}$ is incomplete if $T_{1}$ or $T_{k}$ is a red $P_{3}$. Otherwise, we say $T_{1} \ldots T_{k}$ is complete.

For the remainder of the section, if we refer to a path vwxyz of type B or a path $T_{1} \ldots T_{k}$ of type $\mathrm{C}$, we shall take it as read that $v, w, x, y, z$ and $T_{1}, \ldots, T_{k}$ are as in Definitions 31 and 32 respectively. Note that paths of type $\mathrm{C}$ are well-defined with respect to direction of traversal - if $v_{1} \ldots v_{p}$ is a path of type $\mathrm{C}$, then so is $v_{p} \ldots v_{1}$. 


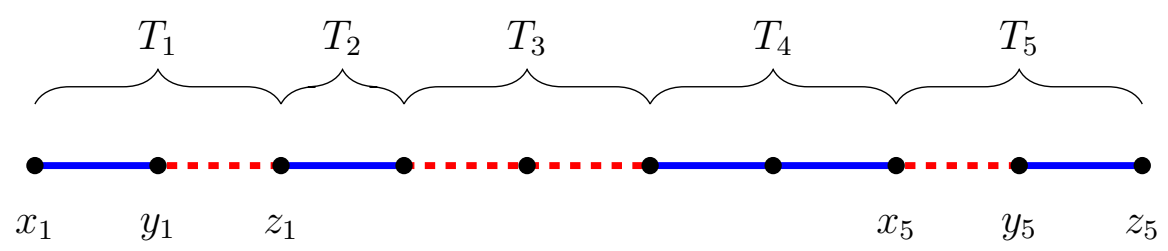

Figure 3: A complete path $T_{1} \ldots T_{5}$ of type C.

See Figure 3 for an example of a path of type C.

We now sketch the proof of Lemma 39. Let $e$ and $f$ be two independent red edges. Using these edges, Builder can force either a path of type B or a path of type $\mathrm{C}$ using Lemma 33. If Builder obtains a path vwxyz of type B, they will apply Lemma 34 to efficiently extend $Q$ using vwxyz.

Suppose instead Builder obtains a path $T_{1} \ldots T_{k}$ of type C. Then we run into a problem $-T_{1} \ldots T_{k}$ is not complete, and only a complete path of type $\mathrm{C}$ may be used to efficiently extend $R$ (see Lemma 38). Builder will therefore use Corollary 37 to extend $T_{1} \ldots T_{k}$ into a path $T_{1}^{\prime} \ldots T_{k^{\prime}}^{\prime}$ of type $\mathrm{C}$ which is either complete or arbitrarily long. Builder then uses Lemma 38 to extend $R$ using $T_{1}^{\prime} \ldots T_{k^{\prime}}^{\prime}$. If $T_{1}^{\prime} \ldots T_{k^{\prime}}^{\prime}$ is complete, this extension is efficient; otherwise, Builder wins the game immediately afterwards by joining $Q$ and the resulting blue path. Thus an incomplete path of type $\mathrm{C}$ is used to extend $R$ at most once over the course of the game, adding only constantly many rounds to the game's length.

We first prove that given two independent red edges Builder can force either a path of type B or a path of type C.

Lemma 33. Given two independent red edges e and $f$, Builder can force Painter to construct one of the following:

(i) a path of type $B$ while uncovering 2 edges;

(ii) an incomplete path $T_{1} T_{2} T_{3}$ of type $C$ and length 5 while uncovering 3 edges;

(iii) a red $P_{4}$ while uncovering 2 edges.

Proof. Write $e=u v$ and $f=x y$. Builder chooses the edges $v w$ and $w x$, where $w$ is a new vertex. If Painter colours both edges red, then $u v w x$ is a red $P_{4}$ and we have achieved (iii). Suppose without loss of generality that Painter colours $v w$ blue. If Painter also colours $w x$ blue, then uvwxy is a path of type B and we have achieved (i). If instead Painter colours $w x$ red, then Builder chooses the edge tu. However Painter colours tu, tuvwxy is now a path of type $\mathrm{C}$ and length 5 , taking $T_{1}=t u v, T_{2}=v w$ and $T_{3}=w x y$. Moreover, $T_{3}$ is a red $P_{3}$, so $T_{1} T_{2} T_{3}$ is incomplete and we have achieved (ii).

We next prove that Builder can use a path of type B to efficiently extend $Q$.

Lemma 34. Suppose $Q$ is a non-trivial blue path with endpoints $a$ and $b$, where $b$ is incident to a red edge bc. Suppose vwxyz is a path of type B vertex-disjoint from $V(Q) \cup$ $\{c\}$. Then, by uncovering at most 3 edges, Builder can force Painter to construct one of the following: 


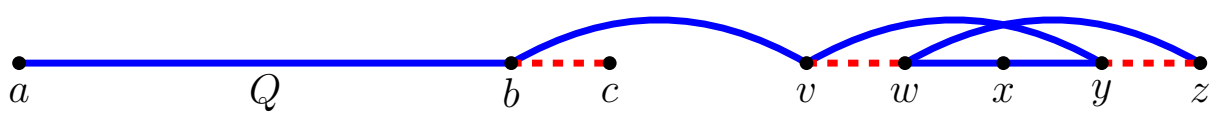

Figure 4: Extending $Q$ using a path of type B as in Lemma 34.

(i) a blue path $Q^{\prime}$ of length $e(Q)+5$ with one endpoint $b^{\prime}$ incident to a red edge $b^{\prime} c^{\prime}$.

(ii) a red $P_{4}$.

Proof. Builder chooses the edges $b v, v y$ and $w z$. If Painter colours any of these edges red, then cbvw, wvyz or vwzy respectively is a red $P_{4}$ and we have achieved (ii). Otherwise, aQbvyxwz is a blue path of length $e(Q)+5$, where $z$ is incident to the red edge $z y$ (see Figure 4), and we have achieved (i).

We now focus on paths of type C. We first note the following simple property of such paths, which follows immediately from their definition (Definition 32).

Proposition 35. Suppose $T_{1} \ldots T_{k}$ is a path of type $C$. Then

$$
e\left(T_{1} \ldots T_{k}\right)=2 k-5+e\left(T_{1}\right)+e\left(T_{k}\right) .
$$

Let $T_{1} \ldots T_{k}$ be an incomplete path of type C. We first prove an ancillary lemma, which says that Builder can always extend an incomplete path of type $\mathrm{C}$ into a slightly longer path of type C.

Lemma 36. Suppose $T_{1} \ldots T_{k}$ is an incomplete path of type $C$ and length $\ell$. Then Builder can force Painter to do one of the following:

(i) for some $i \in\{3,4\}$, extend $T_{1} \ldots T_{k}$ to a path $T_{1}^{\prime} \ldots T_{k+2}^{\prime}$ of type $C$ and length $\ell+i$ while uncovering $i$ edges.

(ii) construct a red $P_{4}$ while uncovering at most 4 edges.

Proof. Suppose without loss of generality that $T_{k}=x_{k} y_{k} z_{k}$ is a red $P_{3}$, where $x_{k} \in$ $V\left(T_{k-1}\right)$. Set $T_{i}^{\prime}=T_{i}$ for $i \leqslant k$. Then Builder chooses two edges $u v$ and $v w$, where $u, v$ and $w$ are new vertices.

First suppose Painter colours both edges blue. Then Builder chooses the edge $z_{k} u$. If Painter colours $z_{k} u$ red, then $x_{k} y_{k} z_{k} u$ is a red $P_{4}$ and we have achieved (ii). If Painter colours $z_{k} u$ blue, then set $T_{k+1}^{\prime}=z_{k} u v$ and $T_{k+2}^{\prime}=v w$. Thus, $T_{1}^{\prime} \ldots T_{k+2}^{\prime}$ is a path of type $\mathrm{C}$ and length $\ell+3$, and we have achieved (i).

Now suppose that Painter colours both $u v$ and $v w$ red. Then Builder chooses the edges $z_{k} t$ and $t u$, where $t$ is a new vertex. If Painter colours one of these edges red, then $x_{k} y_{k} z_{k} t$ or tuvw is a red $P_{4}$, respectively, and we have achieved (ii). If Painter colours both $z_{k} t$ and $t u$ blue, then set $T_{k+1}^{\prime}=z_{k} t u$ and $T_{k+2}^{\prime}=u v w$. Thus, $T_{1}^{\prime} \ldots T_{k+2}^{\prime}$ is a path of type $\mathrm{C}$ and length $\ell+4$, and we have achieved (i). 
Finally, suppose without loss of generality that Painter colours $u v$ blue and $v w$ red. Then Builder chooses the edges $z_{k} u$ and $w x$, where $x$ is a new vertex. If Painter colours $z_{k} u$ red, then $x_{k} y_{k} z_{k} u$ is a red $P_{4}$ and we have achieved (ii). If Painter colours $z_{k} u$ blue, then set $T_{k+1}^{\prime}=z_{k} u v$ and $T_{k+2}^{\prime}=v w x$. Thus $T_{1}^{\prime} \ldots T_{k+2}^{\prime}$ is a path of type $\mathrm{C}$ of length $\ell+4$, however Painter colours $w x$, and we have achieved (i).

By applying Lemma 36 repeatedly, Builder can extend the path $T_{1} T_{2} T_{3}$ of type $\mathrm{C}$ given by Lemma 33 into either a complete path of type $\mathrm{C}$ or an arbitrarily long incomplete path of type C. Recall from Proposition 35 that a path $T_{1} \ldots T_{k}$ of type $\mathrm{C}$ has length at most $2 k-1$.

Corollary 37. Let $k_{0} \geqslant 5$ be an odd integer. Suppose $T_{1} T_{2} T_{3}$ is an incomplete path of type $C$ and length 5. Then Builder can force Painter to do one of the following:

(i) for some $k, \ell \in \mathbb{N}$, extend $T_{1} T_{2} T_{3}$ to a complete path $T_{1}^{\prime} \ldots T_{k}^{\prime}$ of type $C$ and length $\ell$ such that $5 \leqslant k \leqslant k_{0}$, while uncovering $\ell-5$ edges.

(ii) for some $\ell \in \mathbb{N}$, extend $T_{1} T_{2} T_{3}$ to an incomplete path $T_{1}^{\prime} \ldots T_{k_{0}}^{\prime}$ of type $C$ and length $\ell$ while uncovering $\ell-5$ edges.

(iii) construct a red $P_{4}$ while uncovering at most $2 k_{0}-6$ edges.

We next prove that Builder can extend $R$ using a path of type $\mathrm{C}$.

Lemma 38. Suppose $T_{1} \ldots T_{k}$ is a path of type $C$ with $k \geqslant 5$ and $e\left(T_{1} \ldots T_{k}\right)=\ell$. Suppose $R$ is a (possibly trivial) blue path which is vertex-disjoint from $T_{1} \ldots T_{k}$. Then Builder can force Painter to construct one of the following:

(i) a blue path $R^{\prime}$ of length $e(R)+(5 k-7) / 2$ while uncovering $3(k-1) / 2$ edges. This case can only occur if $T_{1} \ldots T_{k}$ is incomplete.

(ii) a blue path $R^{\prime}$ of length $e(R)+\ell^{\prime}$ while uncovering at most $7 \ell^{\prime} / 5-\ell$ edges for some $1 \leqslant \ell^{\prime} \leqslant 5(k-1) / 2$. This case can only occur if $T_{1} \ldots T_{k}$ is complete.

(iii) a red $P_{4}$ while uncovering at most $3(k-1) / 2$ edges.

Proof. Let $a$ and $b$ be the endpoints of $R$. (If $R$ is trivial, then let $a=b$.)For $i \in$ $\{3,5, \ldots, k-2\}$, write $T_{i}=x_{i} y_{i} z_{i}$ where $x_{i} \in V\left(T_{i-1}\right)$ and $z_{i} \in V\left(T_{i+1}\right)$. Thus $x_{i} y_{i} z_{i}$ is a red $P_{3}$ for each $i \in\{3,5, \ldots, k-2\}$. Builder chooses the set

$$
F_{1}=\left\{x_{3} a, b z_{3}, x_{5} c_{1}, c_{1} z_{5}, x_{7} c_{2}, c_{2} z_{7}, \ldots, x_{k-2} \frac{c_{\frac{k-5}{2}}}{2}, c_{\frac{k-5}{2}} z_{k-2}\right\}
$$

of edges, where $c_{1}, \ldots, c_{\frac{k-5}{2}}$ are new vertices. Note that

$$
\left|F_{1}\right|=2+2 \cdot \frac{k-5}{2}=k-3<\frac{3(k-1)}{2} .
$$

If Painter colours an edge in $F_{1}$ red, say $x_{i} w$ or $w z_{i}$ for some integer $i$ and some vertex $w$, then $z_{i} y_{i} x_{i} w$ or $w z_{i} y_{i} x_{i}$ respectively is a red $P_{4}$. So in this case we have achieved (iii). 


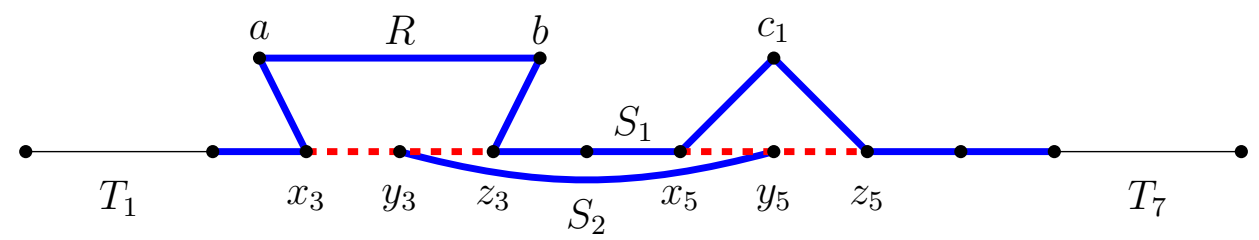

Figure 5: Structure of $S_{1}$ and $S_{2}$ in Lemma 38 for a path $T_{1} \ldots T_{7}$ of type C.

Now suppose Painter colours all edges in $F_{1}$ blue. Then we have obtained a blue path

$$
S_{1}=T_{2} x_{3} a R b z_{3} T_{4} x_{5} c_{1} z_{5} T_{6} x_{7} c_{2} z_{7} \ldots T_{k-3} x_{k-2} \frac{c_{k-5}}{2} z_{k-2} T_{k-1} .
$$

Note that $S_{1}$ has length

$$
\begin{aligned}
e\left(S_{1}\right) & =e\left(T_{2}\right)+e\left(T_{4}\right)+\cdots+e\left(T_{k-1}\right)+\left|F_{1}\right|+e(R) \\
& =\left(2 \cdot \frac{k-3}{2}+1\right)+(k-3)+e(R)=e(R)+2 k-5
\end{aligned}
$$

where the second equality follows from (3).

Builder now chooses the set

$$
F_{2}=\left\{y_{3} y_{5}, y_{5} y_{7}, \ldots, y_{k-4} y_{k-2}\right\}
$$

of edges. Note that $\left|F_{2}\right|=(k-5) / 2$, so by (3) we have uncovered

$$
\left|F_{1}\right|+\left|F_{2}\right|=k-3+\frac{k-5}{2}=\frac{3 k-11}{2}
$$

edges in total so far. If Painter colours an edge in $F_{2}$ red, say $y_{i} y_{i+2}$ for some $i \in$ $\{3,5, \ldots, k-4\}$, then $z_{i} y_{i} y_{i+2} x_{i+2}$ is a red $P_{4}$. So in this case we have achieved (iii). Suppose Painter colours all edges in $F_{2}$ blue. Then we have obtained a blue path

$$
S_{2}=y_{k-2} y_{k-4} \ldots y_{5} y_{3}
$$

Note that $S_{2}$ has length $\left|F_{2}\right|=(k-5) / 2$. Moreover, $S_{1}$ and $S_{2}$ are vertex-disjoint (see Figure 5) and by (4) we have

$$
e\left(S_{1}\right)+e\left(S_{2}\right)=e(R)+2 k-5+\frac{k-5}{2}=e(R)+\frac{5(k-3)}{2} .
$$

Our aim is now to join $S_{1}$ and $S_{2}$ together to form $R^{\prime}$. The way in which we do this depends on the structure of $T_{1}$ and $T_{k}$.

Case 1: $T_{1} \ldots T_{k}$ is incomplete.

Without loss of generality we may assume that $T_{1}$ is a red $P_{3}$, say $x_{1} y_{1} z_{1}$ with $z_{1} \in$ $V\left(T_{2}\right)$. Builder chooses the edges $y_{1} y_{k-2}, y_{3} x_{1}, x_{1} u$ and $u z_{1}$, where $u$ is a new vertex. In total, Builder has uncovered $\left|F_{1}\right|+\left|F_{2}\right|+4=3(k-1) / 2$ edges by (5). If Painter 


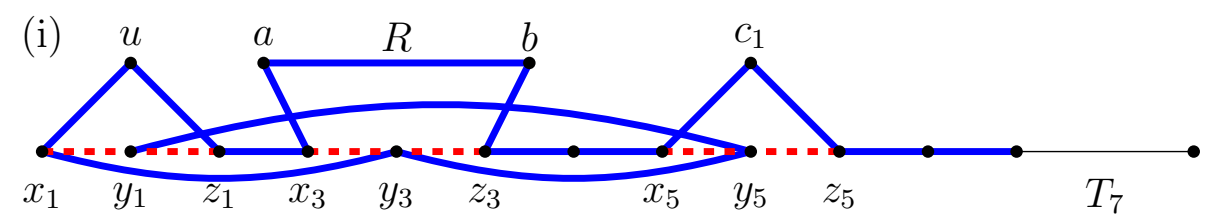

(ii)

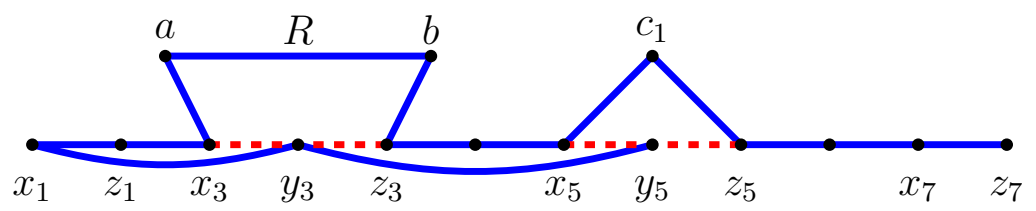

(iii)
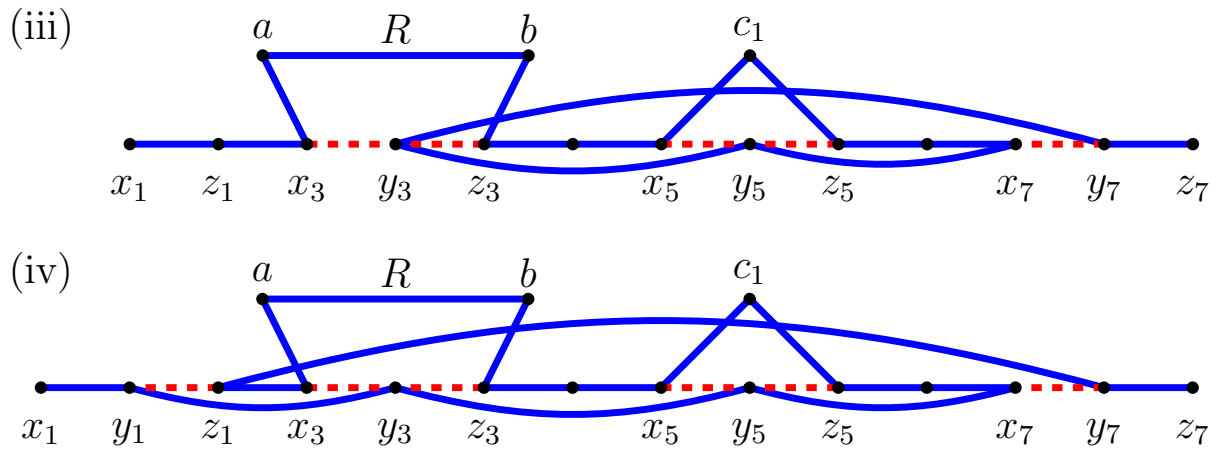

Figure 6: Extending a blue path $R$ with a path $T_{1} \ldots T_{7}$ as in cases 1 through 4 (respectively) of Lemma 38.

colours any of the edges red, then $x_{1} y_{1} y_{k-2} z_{k-2}, y_{3} x_{1} y_{1} z_{1}, z_{1} y_{1} x_{1} u$ or $u z_{1} y_{1} x_{1}$ is a red $P_{4}$, respectively, and we have achieved (iii). Suppose Painter colours them all blue. Then $R^{\prime}:=y_{1} y_{k-2} S_{2} y_{3} x_{1} u z_{1} S_{1}$ is a blue path of length $e\left(S_{1}\right)+e\left(S_{2}\right)+4=e(R)+(5 k-7) / 2$ by (6) (see Figure 6(i)) and hence we have achieved (i).

Case 2: $\quad T_{1} \ldots T_{k}$ is complete and each of $T_{1}$ and $T_{k}$ is a blue edge.

Write $T_{1}=x_{1} z_{1}$ and $T_{k}=x_{k} z_{k}$ with $z_{1} \in V\left(T_{2}\right)$ and $x_{k} \in V\left(T_{k-1}\right)$. First suppose that $k \geqslant 7$. Builder chooses the edges $y_{3} x_{1}$ and $y_{k-2} x_{1}$. In total, Builder has uncovered $\left|F_{1}\right|+\left|F_{2}\right|+2=(3 k-7) / 2$ edges by (5). If Painter colours both edges red, then $x_{3} y_{3} x_{1} y_{k-2}$ is a red $P_{4}$ and we have achieved (iii). Suppose Painter colours $x_{1} y_{3}$ blue. Then $R^{\prime}:=$ $S_{2} y_{3} x_{1} z_{1} S_{1} x_{k} z_{k}$ is a blue path of length $e\left(S_{1}\right)+e\left(S_{2}\right)+3=e(R)+(5 k-9) / 2$ by (6) (see Figure 6(ii)). Writing $\ell^{\prime}:=e\left(R^{\prime}\right)-e(R)=(5 k-9) / 2$, Builder has uncovered

$$
\frac{3 k-7}{2}<\frac{7}{5} \cdot \frac{5 k-9}{2}-(2 k-3)=\frac{7 \ell^{\prime}}{5}-\ell
$$

edges in total, where the last equality follows from Proposition 35. Hence we have achieved (ii). If instead Painter colours $x_{1} y_{k-2}$ blue, the same argument shows we have achieved (ii) on replacing $S_{2} y_{3}$ by $S_{2} y_{k-2}$. So if $k \geqslant 7$, we are done.

If instead $k=5$, Builder chooses the edges $y_{3} x_{1}$ and $u x_{1}$, where $u$ is a new vertex. If Painter colours both edges red, then $u x_{1} y_{3} z_{3}$ is a red $P_{4}$ and we have achieved (iii). Suppose instead Painter colours $w x_{1}$ blue for some $w \in\left\{u, y_{3}\right\}$. Then $R^{\prime}:=w x_{1} z_{1} S_{1} x_{5} z_{5}$ 
is a blue path of length $e\left(S_{1}\right)+e\left(S_{2}\right)+3$ (as $e\left(S_{2}\right)=0$ ) and Builder has uncovered $\left|F_{1}\right|+\left|F_{2}\right|+2$ edges. Thus we have achieved (ii) as above.

Case 3: $T_{1} \ldots T_{k}$ is complete and exactly one of $T_{1}$ and $T_{k}$ is a blue edge.

Without loss of generality we may assume that $T_{1}$ is a blue edge. Let $T_{1}=x_{1} z_{1}$ with $z_{1} \in V\left(T_{2}\right)$, and let $T_{k}=x_{k} y_{k} z_{k}$ with $x_{k} \in V\left(T_{k-1}\right)$. Note that $x_{k} y_{k}$ is red and $y_{k} z_{k}$ is blue. Builder chooses the edges $x_{k} y_{k-2}$ and $y_{3} y_{k}$. In total, Builder has uncovered $\left|F_{1}\right|+\left|F_{2}\right|+2=(3 k-7) / 2$ edges by (5). If Painter colours either $x_{k} y_{k-2}$ or $y_{3} y_{k}$ red, then $y_{k} x_{k} y_{k-2} x_{k-2}$ or $x_{3} y_{3} y_{k} x_{k}$ is a red $P_{4}$ respectively, and we have achieved (iii). Suppose Painter instead colours both edges blue. Then $R^{\prime}:=x_{1} z_{1} S_{1} x_{k} y_{k-2} S_{2} y_{3} y_{k} z_{k}$ is a blue path of length $e\left(S_{1}\right)+e\left(S_{2}\right)+4=e(R)+(5 k-7) / 2$ by (6) (see Figure 6(iii)). Writing $\ell^{\prime}:=e\left(R^{\prime}\right)-e(R)=(5 k-7) / 2$, Builder has uncovered

$$
\frac{3 k-7}{2}<\frac{7}{5} \cdot \frac{5 k-7}{2}-(2 k-2)=\frac{7 \ell^{\prime}}{5}-\ell
$$

edges in total, where the last equality follows from Proposition 35. Hence we have achieved (ii).

Case 4: $T_{1} \ldots T_{k}$ is complete and neither $T_{1}$ nor $T_{k}$ is a blue edge.

Let $T_{1}=x_{1} y_{1} z_{1}$ and $T_{k}=x_{k} y_{k} z_{k}$ where $z_{1} \in V\left(T_{2}\right)$ and $x_{k} \in V\left(T_{k-1}\right)$. Thus $x_{1} y_{1}$ and $y_{k} z_{k}$ are blue, and $y_{1} z_{1}$ and $x_{k} y_{k}$ are red. Then Builder chooses the edges $y_{k} z_{1}$, $x_{k} y_{k-2}$, and $y_{3} y_{1}$. In total, Builder has uncovered $\left|F_{1}\right|+\left|F_{2}\right|+3=(3 k-5) / 2$ edges by (5). If Painter colours one of these edges red, then $x_{k} y_{k} z_{1} y_{1}, y_{k} x_{k} y_{k-2} x_{k-2}$ or $z_{3} y_{3} y_{1} z_{1}$ respectively is a red $P_{4}$ and we have achieved (iii). Suppose Painter colours them all blue. Then $R^{\prime}:=z_{k} y_{k} z_{1} S_{1} x_{k} y_{k-2} S_{2} y_{3} y_{1} x_{1}$ is a blue path (see Figure 6(iv)) of length $e\left(S_{1}\right)+e\left(S_{2}\right)+5=e(R)+5(k-1) / 2$ by $(6)$. Writing $\ell^{\prime}:=e\left(R^{\prime}\right)-e(R)=(5 k-5) / 2$, Builder has uncovered

$$
\frac{3 k-5}{2}=\frac{7}{5} \cdot \frac{5 k-5}{2}-(2 k-1)=\frac{7 \ell^{\prime}}{5}-\ell
$$

edges in total, where the last equality follows from Proposition 35. We have achieved case (ii).

Finally, we consolidate Lemmas 33, 34 and 38 and Corollary 37 into a single lemma which says that given two independent red edges, Builder can extend either $Q$ or $R$. As with Lemma 30, in applying Lemma 39 we will take $m$ to be $\ell-e(Q)-e(R)-1$.

Lemma 39. Let $m \geqslant 9$ be an integer. Let $Q$ and $R$ be blue paths and let e and $f$ be two red edges. Suppose that $Q$ is non-trivial and has an endpoint $b$ incident to a red edge bc. Further suppose that $V(Q) \cup\{c\}, R$, e and $f$ are pairwise vertex-disjoint. Then Builder can force Painter to construct one of the following:

(i) a blue path $Q^{\prime}$ with one endpoint $b^{\prime}$ incident to a red edge $b^{\prime} c^{\prime}$ such that $e\left(Q^{\prime}\right)=$ $e(Q)+5$, while uncovering 5 edges. Moreover, $R$ is vertex-disjoint from $V\left(Q^{\prime}\right) \cup\left\{c^{\prime}\right\}$.

(ii) a blue path $R^{\prime}$ such that $e\left(R^{\prime}\right)=e(R)+\ell^{\prime}$ for some $1 \leqslant \ell^{\prime} \leqslant m+5$ while uncovering at most $7 \ell^{\prime} / 5-2$ edges. Moreover, $R^{\prime}$ is vertex-disjoint from $V(Q) \cup\{c\}$. 
(iii) a blue path $R^{\prime}$ such that $e\left(R^{\prime}\right) \geqslant e(R)+m$ while uncovering at most $7 m / 5+6$ edges. Moreover, $R^{\prime}$ is vertex-disjoint from $V(Q) \cup\{c\}$.

(iv) a red $P_{4}$ while uncovering at most $7 m / 5+6$ edges.

Proof. We first apply Lemma 33 to $e$ and $f$. If as in Lemma 33(iii) we obtain a red $P_{4}$ while uncovering 2 edges, then we have achieved (iv). Suppose we do not. Then we split into cases depending on Painter's choice.

Case 1: We obtain a path vwxyz of type B while uncovering 2 edges, as in Lemma 33(i). Moreover, vwxyz is vertex-disjoint from $V(Q) \cup\{c\}$ and $R$.

We apply Lemma 34 to $Q$ and vwxyz. Hence we have uncovered at most 5 edges in total. If we obtain a red $P_{4}$, then we have achieved (iv). Suppose instead we obtain a blue path $Q^{\prime}$ of length $q+5$ with one endpoint $b^{\prime}$ incident to a red edge $b^{\prime} c^{\prime}$, where $V\left(Q^{\prime}\right) \cup\left\{c^{\prime}\right\}$ is vertex-disjoint from $R$. Then we have achieved (i).

Case 2: We obtain an incomplete path $T_{1} T_{2} T_{3}$ of type $\mathrm{C}$ and length 5 while uncovering 3 edges, as in Lemma 33(ii). Moreover, $T_{1} T_{2} T_{3}$ is vertex-disjoint from $V(Q) \cup\{c\}$ and $R$.

Let $k_{0}$ be the least odd number such that $k_{0} \geqslant(2 m+7) / 5$. Since $5 k_{0}<(2 m+7)+5 \cdot 2$, and both $5 k_{0}$ and $2 m+17$ are odd integers, we have $k_{0} \leqslant 2 m / 5+3$. Moreover, $k_{0} \geqslant$ $(2 m+7) / 5 \geqslant 5$ since $m \geqslant 9$. We apply Corollary 37 to $T_{1} T_{2} T_{3}$ and $k_{0}$. If we obtain a red $P_{4}$ while uncovering at most $2 k_{0}-6$ additional edges, then we have achieved (iv). Suppose we do not. Then we split into subcases depending on Painter's choice.

Case 2a: For some $k, \ell \in \mathbb{N}$, we obtain a complete path $T_{1}^{\prime} \ldots T_{k}^{\prime}$ of type $\mathrm{C}$ and length $\ell$ such that $5 \leqslant k \leqslant k_{0}$ while uncovering $\ell-5$ additional edges, as in Corollary 37(i). Moreover, $T_{1}^{\prime} \ldots T_{k}^{\prime}$ is vertex-disjoint from $V(Q) \cup\{c\}$ and $R$.

We now apply Lemma 38 to $T_{1}^{\prime} \ldots T_{k}^{\prime}$ and $R$. Suppose we obtain a blue path $R^{\prime}$ with length $e(R)+\ell^{\prime}$, where

$$
\ell^{\prime} \leqslant \frac{5(k-1)}{2} \leqslant \frac{5\left(k_{0}-1\right)}{2} \leqslant \frac{5}{2} \cdot\left(\frac{2 m}{5}+2\right)=m+5,
$$

while uncovering at most $7 \ell^{\prime} / 5-\ell$ edges as in Lemma 38(ii). Note that $R^{\prime}$ is vertex-disjoint from $V(Q) \cup\{c\}$. In total we have uncovered at most $3+(\ell-5)+\left(7 \ell^{\prime} / 5-\ell\right)=7 \ell^{\prime} / 5-2$ edges, so we have achieved (i).

Suppose instead we obtain a red $P_{4}$ while uncovering at most $3(k-1) / 2$ edges as in Lemma 38(iii). Note that $\ell \leqslant 2 k_{0}-1$ by Proposition 35. In total we have therefore uncovered at most

$$
3+(\ell-5)+\frac{3\left(k_{0}-1\right)}{2} \leqslant \frac{7 k_{0}-9}{2} \leqslant \frac{7}{2} \cdot\left(\frac{2 m}{5}+3\right)-\frac{9}{2}=\frac{7 m}{5}+6
$$

edges, and thus we have achieved (iv).

Case 2b: For some $\ell \in \mathbb{N}$, we obtain an incomplete path $T_{1}^{\prime} \ldots T_{k_{0}}^{\prime}$ of type $\mathrm{C}$ and length $\ell$ while uncovering $\ell-5$ additional edges, as in Corollary 37(ii). Moreover, $T_{1}^{\prime} \ldots T_{k_{0}}^{\prime}$ is vertex-disjoint from $V(Q) \cup\{c\}$ and $R$. 
We apply Lemma 38 to $T_{1}^{\prime} \ldots T_{k_{0}}^{\prime}$ and $R$. Whatever the outcome, we uncover at most $3\left(k_{0}-1\right) / 2$ edges. We have therefore uncovered at most $7 \mathrm{~m} / 5+6$ edges in total, as in $(7)$. If we obtain a red $P_{4}$ as in Lemma 38(iii), then we have achieved (iv). Hence we may assume that we obtain a blue path $R^{\prime}$ of length

$$
e(R)+\frac{5 k_{0}-7}{2} \geqslant e(R)+\frac{5}{2} \cdot \frac{2 m+7}{5}-\frac{7}{2}=e(R)+m
$$

as in Lemma 38(i). (The inequality follows from the definition of $k_{0}$.) We have therefore achieved (iii).

\section{A.3 An upper bound on $\tilde{\boldsymbol{r}}\left(\boldsymbol{P}_{4}, \boldsymbol{P}_{\ell+1}\right)$ for $\ell \geqslant 3$}

We now use Lemmas 26,30 and 39 to bound $\tilde{r}\left(P_{4}, P_{\ell+1}\right)$ above in Theorem 44 . Together with Theorem 4, this will imply Theorem 6 .

Recall that Builder's strategy is to extend blue paths $Q$ and $R$ using independent edges. For the remainder of the section, we denote the graph Builder has uncovered by $G$. In order to keep track of the lengths of $Q$ and $R$ and the number of independent edges available, we introduce the following notation.

Definition 40. Let $G$ be a graph. Given $q, r, n_{\text {blue }}, n_{\text {red }} \in \mathbb{N}_{0}$, we say that $G$ contains a $\left(q, r, n_{\text {blue }}, n_{\text {red }}\right)$-structure if it satisfies the following properties:

(P1) $G$ contains a (possibly trivial) blue path $Q$ of length $q$ with one endpoint $b$ incident to a red edge $b c$.

(P2) $G$ contains a (possibly trivial) blue path $R$ of length $r$.

(P3) $G$ contains a set $F$ of independent edges containing $n_{\text {blue }}$ blue edges and $n_{\text {red }}$ red edges.

(P4) $V(Q) \cup\{c\}, R$ and $F$ are pairwise vertex-disjoint.

This notation substantially simplifies the statements of Lemmas 26, 30 and 39. The corresponding statements are as follows.

Corollary 41. Let $q, r, n_{\text {red }}, n_{\text {blue }} \in \mathbb{N}_{0}$ and let $G$ be a graph. Suppose $G$ contains a $\left(q, r, n_{\text {blue }}, n_{\text {red }}\right)$-structure. Then Builder can force Painter to construct a graph $G^{\prime} \supseteq G$ with $e\left(G^{\prime}\right) \leqslant e(G)+2$ such that $G^{\prime}$ contains a $\left(q+r+1,0, n_{\text {blue }}, n_{\text {red }}\right)$-structure or a red $P_{4}$.

Corollary 42. Let $m, q, r, n_{\mathrm{red}} \in \mathbb{N}_{0}$ with $q, m \geqslant 1$. Suppose $G$ is a graph containing a $\left(q, r, 2, n_{\text {red }}\right)$-structure. Then Builder can force Painter to construct a graph $G^{\prime} \supseteq G$ such that one of the following holds:

(i) $G^{\prime}$ contains a $\left(q+\ell^{\prime}, r, n_{\text {blue }}, n_{\text {red }}\right)$-structure and $e\left(G^{\prime}\right)=e(G)+\ell^{\prime}$ for some $3 \leqslant$ $\ell^{\prime} \leqslant m+3$ and some $n_{\text {blue }} \in \mathbb{N}_{0}$. Moreover, if $3 \leqslant \ell^{\prime}<5 \leqslant m$, then we may take $n_{\text {blue }}=1$. 
(ii) $G^{\prime}$ contains a red $P_{4}$ and $e\left(G^{\prime}\right) \leqslant e(G)+m+3$.

Corollary 43. Let $m, q, r, n_{\text {blue }} \in \mathbb{N}_{0}$ with $q \geqslant 1$ and $m \geqslant 9$. Suppose $G$ is a graph containing a $\left(q, r, n_{\text {blue }}, 2\right)$-structure. Then Builder can force Painter to construct a graph $G^{\prime} \supseteq G$ such that one of the following holds:

(i) $e\left(G^{\prime}\right)=e(G)+5$ and $G^{\prime}$ contains a $\left(q+5, r, n_{\text {blue }}, 0\right)$-structure.

(ii) There exists $1 \leqslant \ell^{\prime} \leqslant m+5$ such that $e\left(G^{\prime}\right) \leqslant e(G)+7 \ell^{\prime} / 5-2$ and $G^{\prime}$ contains a $\left(q, r+\ell^{\prime}, n_{\text {blue }}, 0\right)$-structure.

(iii) $e\left(G^{\prime}\right) \leqslant e(G)+7 m / 5+6$ and $G^{\prime}$ contains a $\left(q, r+m, n_{\text {blue }}, 0\right)$-structure.

(iv) $e\left(G^{\prime}\right) \leqslant e(G)+7 m / 5+6$ and $G^{\prime}$ contains a red $P_{4}$.

Theorem 44. For all $\ell \in \mathbb{N}$, we have $\tilde{r}\left(P_{4}, P_{\ell+1}\right) \leqslant(7 \ell+52) / 5$.

Proof. Our aim is to show that Builder can construct a graph $G$ with $e(G) \leqslant(7 \ell+52) / 5$ containing a red $P_{4}$ or a blue $P_{\ell+1}$.

We first obtain an initial blue path $Q$ with one endpoint incident to a red edge. We claim that either Builder can construct a path $x y S z$ of type A with $e(S)<\ell$, while uncovering at most $(7 e(S)+4) / 5$ edges, or we are done. We proceed as follows. Builder chooses an edge $e=u v$. First suppose Painter colours $u v$ blue. Then apply Lemma 28 to $u v$, taking $m=\ell$. If we find a blue $P_{\ell+1}$ while uncovering $\ell-1$ additional edges, then since we have uncovered $\ell$ edges in total we are done. Suppose instead we find a path $x y S z$ of type A with $e(S)<\ell$, while uncovering $e(S)$ additional edges in the process. Then in total Builder has uncovered $e(S)+1<(7 e(S)+4) / 5$ edges, as desired.

Suppose instead Painter colours $u v$ red. Then Builder chooses the edge $v x$, where $x$ is a new vertex. If Painter colours $v x$ blue, then $u v x$ is a path of type A constructed while uncovering $2<(7+4) / 5$ edges in total. If Painter colours $v x$ red, then Builder chooses the edges $t u, u w$ and $w x$, where $t$ and $w$ are new vertices. If Painter colours any of these edges red, then tuvx, xvuw or wxvu respectively is a red $P_{4}$ and we are done. Otherwise, tuwxv is a path of type A (taking $S=t u w x$ ), constructed while uncovering $5=(7 \cdot 3+4) / 5$ edges in total. Therefore, we may assume that Builder has constructed a path $x y S z$ of type A with $e(S)<\ell$ while uncovering at most $(7 e(S)+4) / 5$ edges as claimed.

Let $G_{0}$ be the graph consisting of all edges uncovered so far. Thus $G_{0}$ contains a $\left(q_{0}, 0,0,0\right)$-structure for some $1 \leqslant q_{0}<\ell$, and $e\left(G_{0}\right) \leqslant\left(7 q_{0}+4\right) / 5$. Suppose that for some $i \geqslant 0$, Builder has already constructed a graph $G_{i}$ such that there exist $q_{i}, r_{i}, n_{\text {blue }, i}, n_{\text {red }, i} \in$ $\mathbb{N}_{0}$ satisfying the following properties:

(G1) $G_{i} \subseteq K_{\mathbb{N}}$ is the graph of all uncovered edges.

(G2) $G_{i}$ contains a $\left(q_{i}, r_{i}, n_{\text {blue }, i}, n_{\text {red }, i}\right)$-structure, and $q_{i}>0$.

(G3) $q_{i}+r_{i} \leqslant \ell+4$. 
(G4) $n_{\text {red }, i}, n_{\text {blue }, i} \leqslant 1$.

(G5) $e\left(G_{i}\right) \leqslant\left(7\left(q_{i}+r_{i}\right)+4\right) / 5+n_{\text {blue }, i}+n_{\text {red }, i}$.

Note that (G1)-(G5) hold for $i=0$. We are going to show that Builder can force a graph $G_{i+1} \supseteq G_{i}$ such that one of the following holds:

(a) $G_{i+1}$ contains a red $P_{4}$ or a blue $P_{\ell+1}$ and $e\left(G_{i+1}\right) \leqslant(7 \ell+52) / 5$.

(b) there exist $q_{i+1}, r_{i+1}, n_{\text {blue }, i+1}, n_{\text {red }, i+1} \in \mathbb{N}_{0}$ such that $q_{i+1}+r_{i+1}>q_{i}+r_{i}$ and $G_{i+1}$, $q_{i+1}, r_{i+1}, n_{\text {blue }, i+1}$ and $n_{\text {red }, i+1}$ together satisfy (G1)-(G5).

If (a) holds, we are done. If (b) holds, then Builder can repeat the algorithm to obtain $G_{i+2}$. We then simply repeat the process until it terminates, which must happen by (G3) (since $q_{i+1}+r_{i+1}>q_{i}+r_{i}$ whenever these quantities are defined). It therefore remains only to prove that forcing such a graph is possible.

Let $m=\ell-q_{i}-r_{i}-1$. We split into cases depending on the values of $q_{i}, r_{i}, n_{\text {blue }, i}$ and $n_{\text {red }, i}$.

Case 1: $q_{i}+r_{i} \geqslant \ell-1$.

In this case, we may simply join our two blue paths together to achieve (a). Apply Corollary 41 to $G_{i}$. Builder obtains a graph $G_{i+1} \supseteq G_{i}$ with

$$
e\left(G_{i+1}\right)=e\left(G_{i}\right)+2 \stackrel{(\mathrm{G} 5)}{\leqslant} \frac{7\left(q_{i}+r_{i}\right)+4}{5}+n_{\text {blue }, i}+n_{\text {red }, i}+2 \stackrel{(\mathrm{G} 3),(\mathrm{G} 4)}{\leqslant} \frac{7 \ell+52}{5} .
$$

Moreover, $G^{\prime}$ contains a red $P_{4}$ or a blue $P_{\ell+1}$, so we have achieved (a).

Case 2: $\quad \ell-9 \leqslant q_{i}+r_{i} \leqslant \ell-2$, so that $1 \leqslant m \leqslant 8$.

In this case, it is more efficient to naively extend our paths to the right combined length and join them than it is to apply our normal extension methods and potentially end up with paths longer than we need. Builder will force a red $P_{4}$ or a blue $P_{\ell+1}$ as follows. Apply Corollary 41 to $G_{i}$ to obtain a graph $G^{\prime} \supseteq G_{i}$ with $e\left(G^{\prime}\right)=e\left(G_{i}\right)+2$. Note that $G^{\prime}$ contains a red $P_{4}$ or a $\left(q_{i}+r_{i}+1,0, n_{\text {blue }, i}, n_{\text {red }, i}\right)$-structure. By repeating the process at most $m$ additional times, Builder obtains a graph $G^{\prime \prime} \supseteq G^{\prime} \supseteq G_{i}$, where

$$
\begin{aligned}
& e\left(G^{\prime \prime}\right) \leqslant e(G)+2 m+2 \stackrel{(\mathrm{G} 5)}{\leqslant} \frac{7\left(q_{i}+r_{i}\right)+4}{5}+n_{\text {blue }, i}+n_{\text {red }, i}+2 m+2 \\
& \stackrel{\text { (G4) }}{\leqslant} \frac{7(\ell-m-1)+4}{5}+2+2 m+2=\frac{7 \ell}{5}+\frac{3 m+17}{5} \leqslant \frac{7 \ell+41}{5},
\end{aligned}
$$

such that $G^{\prime \prime}$ contains a red $P_{4}$ or a $\left(q_{i}+r_{i}+m+1,0, n_{\text {blue }, i}, n_{\text {red }, i}\right)$-structure (which contains a blue $P_{\ell+1}$ ). Thus we have achieved (a).

Case 3: $q_{i}+r_{i} \leqslant \ell-10$, so that $m \geqslant 9$.

In this case, we will extend our paths efficiently using Corollaries 42 and 43. By choosing at most $3-n_{\text {blue }, i}-n_{\text {red }, i}$ additional independent edges (on new vertices), Builder 
obtains a graph $G_{i}^{\prime} \supseteq G_{i}$ containing a $\left(q_{i}, r_{i}, n_{\text {blue }}^{\prime}, n_{\text {red }}^{\prime}\right)$-structure such that $n_{\text {blue }}^{\prime}+n_{\text {red }}^{\prime} \leqslant 3$, either $n_{\text {blue }}^{\prime}=2$ or $n_{\text {red }}^{\prime}=2$, and

$$
e\left(G_{i}^{\prime}\right) \stackrel{(\mathrm{G} 5)}{\leqslant} \frac{7\left(q_{i}+r_{i}\right)+4}{5}+n_{\text {blue }}^{\prime}+n_{\text {red }}^{\prime} .
$$

We split into subcases depending on the values of $n_{\text {blue }}^{\prime}$ and $n_{\text {red }}^{\prime}$.

Case 3a: $n_{\text {blue }}^{\prime}=2$ and $n_{\text {red }}^{\prime} \leqslant 1$.

We apply Corollary 42 to $G_{i}^{\prime}$, obtaining a graph $G^{\prime} \supseteq G_{i}^{\prime}$. First suppose Corollary 42(i) holds, so that there exists some $3 \leqslant \ell^{\prime} \leqslant m+3$ such that $G^{\prime}$ contains a $\left(q_{i}+\ell^{\prime}, r_{i}, n_{\text {blue }}^{\prime \prime}, n_{\text {red }}^{\prime}\right)$ structure and $e\left(G^{\prime}\right)=e\left(G_{i}^{\prime}\right)+\ell^{\prime}$. Set $G_{i+1}=G^{\prime}, q_{i+1}=q_{i}+\ell^{\prime}, r_{i+1}=r_{i}$ and $n_{\mathrm{red}, i+1}=n_{\mathrm{red}}^{\prime}$. Set $n_{\text {blue }, i+1}=0$ if $\ell^{\prime} \geqslant 5$ and $n_{\text {blue }, i+1}=1$ otherwise. Clearly $q_{i+1}+r_{i+1}>q_{i}+r_{i}$, and (G1) and (G4) are satisfied. Recall from Corollary 42(i) that if $\ell^{\prime}<5 \leqslant m$ then we may take $n_{\text {blue }}^{\prime \prime}=1$, so (G2) is satisfied. We have $q_{i+1}+r_{i+1} \leqslant q_{i}+m+3+r_{i}=\ell+2$, so (G3) is satisfied. If $3 \leqslant \ell^{\prime} \leqslant 4$, we have

$$
\begin{aligned}
e\left(G^{\prime}\right) & =e\left(G_{i}^{\prime}\right)+\ell^{\prime} \stackrel{(8)}{\leqslant} \frac{7\left(q_{i}+r_{i}\right)+4}{5}+2+n_{\mathrm{red}}^{\prime}+\ell^{\prime} \\
& =\frac{7\left(q_{i}+r_{i}+\ell^{\prime}\right)+4}{5}-\frac{2 \ell^{\prime}}{5}+2+n_{\text {red }}^{\prime} \leqslant \frac{7\left(q_{i+1}+r_{i+1}\right)+4}{5}+1+n_{\text {red }}^{\prime} \\
& =\frac{7\left(q_{i+1}+r_{i+1}\right)+4}{5}+n_{\text {blue }, i+1}+n_{\text {red }, i+1} .
\end{aligned}
$$

So (G5) is satisfied and we have therefore achieved (b). A similar argument holds for the case when $\ell^{\prime} \geqslant 5$.

Suppose instead that Corollary 42(ii) holds, so that $G^{\prime}$ contains a red $P_{4}$ and $e\left(G^{\prime}\right) \leqslant$ $e\left(G_{i}^{\prime}\right)+m+3$. Then we have

$$
e\left(G^{\prime}\right) \stackrel{(8)}{\leqslant} \frac{7\left(q_{i}+r_{i}\right)+4}{5}+2+n_{\mathrm{red}}^{\prime}+m+3 \leqslant \frac{2\left(q_{i}+r_{i}\right)+4}{5}+\ell+5 \leqslant \frac{7 \ell+9}{5},
$$

where the final inequality follows since $q_{i}+r_{i} \leqslant \ell-10$. We have therefore achieved (a).

Case 3b: $n_{\text {red }}^{\prime}=2$ and $n_{\text {blue }}^{\prime} \leqslant 1$.

We apply Corollary 43 to $G_{i}^{\prime}$, obtaining a graph $G^{\prime} \supseteq G_{i}^{\prime}$. Suppose Corollary 43(i) or (ii) holds. In either case, it follows that there exist $q^{\prime}$ and $r^{\prime}$ such that $G^{\prime}$ contains a $\left(q^{\prime}, r^{\prime}, n_{\text {blue }}^{\prime}, 0\right)$-structure and

$$
1 \leqslant q^{\prime}+r^{\prime}-\left(q_{i}+r_{i}\right) \leqslant m+5
$$

Write $\ell^{\prime}=q^{\prime}+r^{\prime}-\left(q_{i}+r_{i}\right)$. Set $G_{i+1}=G^{\prime}, q_{i+1}=q^{\prime}, r_{i+1}=r^{\prime}, n_{\text {blue }, i+1}=n_{\text {blue }}^{\prime}$ and $n_{\mathrm{red}, i+1}=0$. Clearly (G1) $-(\mathrm{G} 4)$ are satisfied, and $q_{i+1}+r_{i+1}>q_{i}+r_{i}$. Moreover, we have

$$
\begin{aligned}
e\left(G_{i+1}\right) & \leqslant e\left(G_{i}^{\prime}\right)+\frac{7 \ell^{\prime}}{5}-2 \stackrel{(8)}{\leqslant} \frac{7\left(q_{i}+r_{i}+\ell^{\prime}\right)+4}{5}+n_{\text {blue }}^{\prime} \\
& =\frac{7\left(q_{i+1}+r_{i+1}\right)+4}{5}+n_{\text {blue }, i+1}+n_{\text {red }, i+1}
\end{aligned}
$$


so (G5) is satisfied. We have therefore achieved (b).

Now suppose Corollary 43(iii) holds, so that $G^{\prime}$ contains a $\left(q_{i}, r_{i}+m, n_{\text {blue }}^{\prime}, 0\right)$-structure and $e\left(G^{\prime}\right) \leqslant e\left(G_{i}^{\prime}\right)+7 m / 5+6$. We apply Corollary 41 to $G^{\prime}$, obtaining a graph $G^{\prime \prime}$ such that

$$
\begin{aligned}
e\left(G^{\prime \prime}\right) & =e\left(G^{\prime}\right)+2 \leqslant e\left(G_{i}^{\prime}\right)+\frac{7 m}{5}+8 \\
& \stackrel{(8)}{\leqslant} \frac{7\left(q_{i}+r_{i}+m\right)+4}{5}+n_{\text {blue }}^{\prime}+10 \leqslant \frac{7 \ell+52}{5} .
\end{aligned}
$$

Moreover, $G^{\prime \prime}$ contains a red $P_{4}$ or an $\left(\ell, 0, n_{\text {blue }}^{\prime}, 0\right)$-structure (which contains a blue $\left.P_{\ell+1}\right)$. We have therefore achieved (a).

Finally suppose Corollary $43(\mathrm{iv})$ holds, so that $G^{\prime}$ contains a red $P_{4}$ and $e\left(G^{\prime}\right) \leqslant$ $e\left(G_{i}^{\prime}\right)+7 m / 5+6$. Then we have

$$
e\left(G^{\prime}\right) \leqslant e\left(G_{i}^{\prime}\right)+\frac{7 m}{5}+6 \stackrel{(8)}{\leqslant} \frac{7\left(q_{i}+r_{i}+m\right)+4}{5}+n_{\text {blue }}^{\prime}+8 \leqslant \frac{7 \ell+42}{5} .
$$

We have therefore achieved (a). This completes the proof of the theorem. 